\title{
Pavement degradation: a city-scale model for San Francisco, USA
}

\section{Bingyu Zhao BEng}

PhD student, Department of Engineering, University of Cambridge,

Cambridge, UK (corresponding author: bz247@cam.ac.uk)

(Orcid:0000-0002-2369-7731)

Elisabete Silva PhD

Reader, Department of Land Economy, University of Cambridge,

Cambridge, UK (Orcid:0000-0002-5816-6447)
Kenichi Soga FREng, FICE

Chancellor's Professor, Department of Civil and Environmental Engineering, University of California-Berkeley, Berkeley, CA, USA

(Orcid:0000-0001-5418-7892)

Data from long-term systematic pavement condition surveys provide opportunities to understand better the pavement degradation process. To provide more accurate predictions on future pavement conditions, spatial conditions are incorporated into degradation models of pavements in this paper. Long-term, city-scale pavement condition data from the San Francisco open data portal in USA are used to test and guide model development. Spatial and non-spatial degradation models are developed and compared with parameter estimations carried out using the Bayesian approach. Specifically, the integrated nested Laplace approximation method is used for the Bayesian regression. It was found that (a) the non-spatial model including only coarse categories of pavement types is too simple to provide a good fit to the data; $(b)$ for models with fine categories (individual street segments), the spatial model is more preferable than the non-spatial model due to its lower deviance information criterion and slightly smaller fitting and testing errors; and (c) only the spatial model can reveal the spatial clustering of streets where high/low degradation rates concentrate.

\section{Notation}

$D \quad$ deviance, a goodness-of-fit statistic

$n_{i} \quad$ number of adjacent streets of street $i$

$p_{D} \quad$ effective number of parameters, a measure of model complexity in the calculation of the deviance information criterion the spatially unstructured part of the street-specific variation in the pavement degradation rate from the average

$\boldsymbol{v} \quad$ the spatially structured part of the street-specific variation in the pavement degradation rate from the average

$\boldsymbol{x} \quad$ age; covariate

$\boldsymbol{y} \quad$ observed Pavement Condition Index (PCI)

$\alpha \quad$ average initial pavement condition; intercept

$\beta \quad$ PCI degradation rate; slope

$\boldsymbol{\eta} \quad$ linear predictor based on covariates

$\boldsymbol{\theta} \quad$ list of model parameters

$\xi \quad$ street-specific variation in initial pavement condition from the average

$\tau_{g} \quad$ precision (inverse of variance) of the outcome variable $y$

$\tau_{u}, \tau_{v}$ precision (inverse of variance) of street-specific variation in degradation rates $u$ and $v$

$\tau_{\alpha} \quad$ precision (inverse of variance) of the intercept variable $\alpha$ $\tau_{\beta} \quad$ precision (inverse of variance) of the slope variable $\beta$

$\tau_{\xi} \quad$ precision (inverse of variance) of street-specific variation in intercept $\xi$

$\psi \quad$ list of model hyperparameters

\section{Introduction}

Thanks to the recent advancements in pavement condition monitoring and management, pavement condition data are becoming available at increasingly large spatial scales and high spatial resolutions (Benbow et al., 2017; Spong and Cartwright, 2017; Wang et al., 2014; Zhao and Nagayama, 2017). This provides both opportunities and challenges for pavement management: the opportunities are to understand network-wide condition change and maintenance needs at high spatio-temporal resolution, while the challenges are to analyse large amounts of spatio-temporal data in an efficient manner and identify meaningful and usable quantifications for pavement maintenance management.

Spatial and spatio-temporal data are widely used in geographic and urban-related studies, such as ecology, meteorology, criminology, land use and transportation (Aljoufie et al., 2013; Baller et al., 2001; Handcock and Wallis, 1994; Lichstein et al., 2002; Silva and Clarke, 2005). In pavement management, there are also works addressing various spatial aspects and proposing methods for visualisation, spatial character quantification, missing data imputation and decision-making (Chen et al., 2014; Saliminejad and Gharaibeh, 2012). But direct applications of spatial and spatio-temporal modelling for infrastructure management are still limited (Anyala et al., 2014; Deshmukh, 2010; Ortiz-García et al., 2006).

This paper incorporates both spatial and temporal dimensions into pavement degradation modelling. It is organised as follows: first, the background is introduced about the necessity of system-wide understanding from pavement asset management perspective. Next, data from the case study area are presented, together with descriptions of the data cleaning procedures. In Section 4, the fundamentals of spatial models are reviewed and a multilevel 
spatial pavement degradation model is proposed. Also in this section, the integrated nested Laplace approximation (INLA) is introduced, which is adopted for parameter inference in this study due to its computational efficiency (Rue et al., 2009). Two nonspatial models and one spatial model with spatially correlated degradation rates are designed to represent a variety of pavement degradation modelling strategies. The results of these three models are compared in terms of their fitting and predicting abilities, the deviance information criterion (DIC) and the spatial representations. Although pavement condition is modelled as degrading linearly with age, this can be modified in the future to include higher-order terms. The aim of this work is to demonstrate the technique, as well as to provide pros and cons of spatial pavement degradation models, which future studies can benefit from when choosing between alternative modelling strategies. The paper is concluded with discussions about the values and limitations of this study and plans for future work.

\section{Background}

Pavement asset managers have always been seeking methods that help with the decisions of when and where to carry out maintenance (Ferreira et al., 2002; Gao and Zhang, 2012; Golabi et al., 1982; Wu et al., 2008; Zhang et al., 2012). In the past, such decisions were largely hindered by the scarcity of data: usually pavement performance models or insights were based on data collected at a small scale and thus not representative enough given the natural variability of the pavement degradation process (Johnson and Cation, 1992; Nunez and Shahin, 1986). The situation has improved recently as, in many places, pavement inspections are carried out more frequently system-wide (Haider et al., 2010; McQueen and Timm, 2005; Sadeghi et al., 2017). However, when it comes to maintenance planning, there are still many difficulties in producing a reliable pavement condition prediction model, particularly with the strong presence of measurement errors inherent to visual surveys and the lack of knowledge on crucial degradation-affecting factors (e.g. construction quality, history of minor maintenance activities), as encountered in this study.

As a result, to address the issue of 'imperfect data', additional structures in the data should be considered as useful information, which will hopefully bring about more insights. There have been several studies incorporating the underlying hierarchies of the pavement degradation process. For example, a model is proposed by Anyala et al. (2014) to assess the impact of climate change on pavement rutting. In this hierarchical Bayesian model, level 1 parameters govern the degradation process of each surface group while at the same time being constrained by level 2 parameters (network level). This hierarchical structure is used to reduce the parameter estimation uncertainties. In another study (Alaswadko et al., 2019), roughness (IRI) was modelled in a linear hierarchical manner, reflecting the structured variations of pavement IRI by each section, highway and road classes. These existing studies mainly rely on the known hierarchical structures of the street network as additional information, while, in this study, it is shown that the similar hierarchical modelling approach can be applied in a more general manner, taking advantage of the natural spatial structures of the street network.

\section{Data}

The pavement condition data used in this study are published by the San Francisco Department of Public Works (SF DPW) on DataSF (2019) under the Open Data Commons Public Domain Dedication and License (ODC, 2009). It provides historical and current information on the Pavement Condition Index (PCI) of more than 12000 street segments in the city (Figure 1). PCI is a numerical scale from 0 to 100 that is used to represent the general condition of pavement, with 0 being badly deteriorated roads and 100 representing brand new conditions. It was originated in the USA in the late 1970s and is still widely used

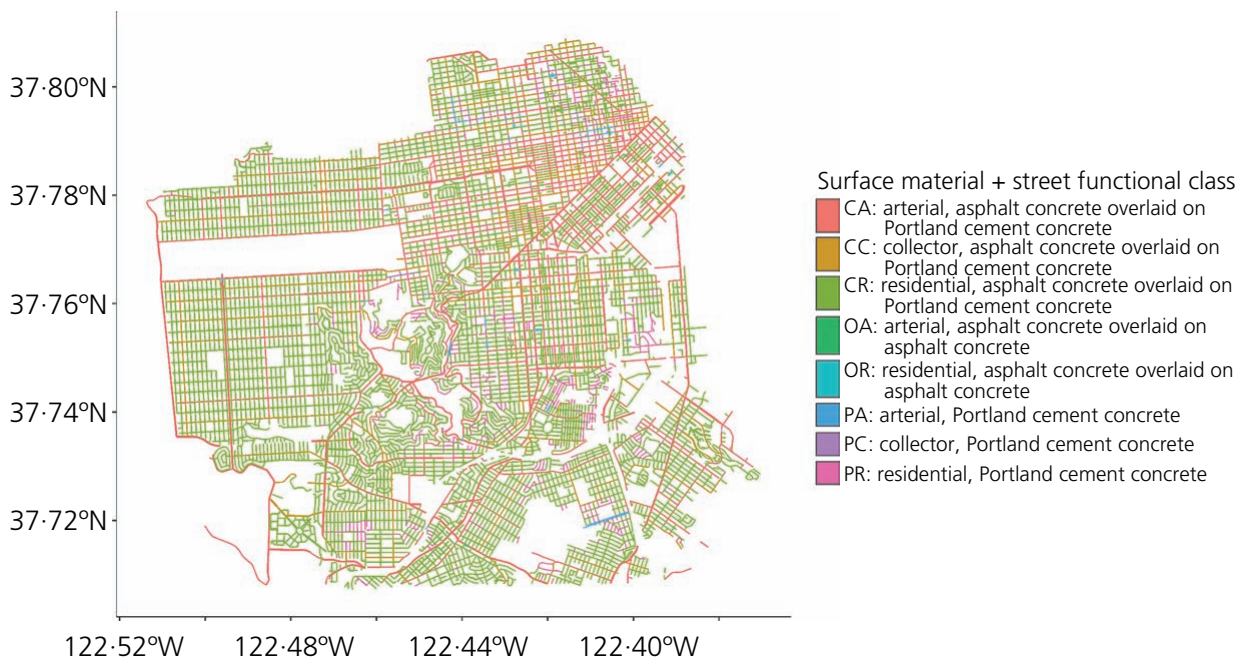

Figure 1. A diagram of the street network in San Francisco, coloured by surface type and functional class categories 
Table 1. Pavement condition records of the 1st Street, between Market Street and Stevenson Street

\begin{tabular}{|c|c|c|c|c|c|c|c|c|}
\hline CNN & $\begin{array}{l}\text { Street } \\
\text { name }\end{array}$ & $\begin{array}{l}\text { From } \\
\text { street }\end{array}$ & To street & $\begin{array}{l}\text { Functional } \\
\text { class }\end{array}$ & $\begin{array}{l}\text { Surface } \\
\text { type }\end{array}$ & $\begin{array}{l}\text { PCI } \\
\text { score }\end{array}$ & $\mathrm{PCl}$ change date & $\begin{array}{l}\text { Maintenance or } \\
\text { survey }\end{array}$ \\
\hline 100000 & 01ST ST & Market St & Stevenson St & Arterial & C & 100 & 09/05/2001 12.00 .00 a.m. & Treatment \\
\hline 100000 & 01ST ST & Market St & Stevenson St & Arterial & C & 100 & 19/11/2002 12.00 .00 a.m. & Survey \\
\hline 100000 & 01ST ST & Market St & Stevenson St & Arterial & C & 100 & 16/11/2005 4.50.06 p.m. & Survey \\
\hline 100000 & $01 \mathrm{ST}$ ST & Market St & Stevenson St & Arterial & C & 100 & 26/07/2007 4.21.27 p.m. & Survey \\
\hline 100000 & 01ST ST & Market St & Stevenson St & Arterial & C & 93 & 10/08/2009 3.43.09 p.m. & Survey \\
\hline 100000 & 01ST ST & Market St & Stevenson St & Arterial & C & 73 & 23/12/2010 1.37.36 p.m. & Survey \\
\hline 100000 & 01ST ST & Market St & Stevenson St & Arterial & C & 56 & 08/01/2013 3.18.02 p.m. & Survey \\
\hline 100000 & 01ST ST & Market St & Stevenson St & Arterial & C & 61 & 25/11/2014 02.17.39 a.m. & Survey \\
\hline
\end{tabular}

for pavement condition assessment (Shahin and Kohn, 1982; Shahin et al., 1978).

Table 1 offers a glimpse of the data set by showing the records belonging to street segment 'CNN100000' at different times. The pavement condition data are collected by the SF DPW using visual surveys, and they have been used for asset management, decision-making and publicity purposes. For example, they are used to demonstrate the pavement condition changes on a yearly basis for each city and county in the Bay Area (Vital Signs, 2017) and to assess the outcome of major infrastructure investments (SF Public Works, 2017). As for pavement performance forecasting, the data are most notably used in the calibration of the pavement performance model in StreetSaver, a pavement management software developed by the Bay Area Metropolitan Transport Commission (MTC) and used by many local agencies in the west coast of the USA. The StreetSaver model utilises a family of deterministic S-shaped curves to predict pavement deterioration as a function of time, with model parameters obtained from weighted least squares regression (Deshmukh, 2010). To improve further the deterministic model in StreetSaver, Ramirez-Flores and Chang-Albitres (2012) proposed a stochastic model that projects pavement conditions as a probability distribution. However, both of these two existing studies categorised the city-scale data by pavement types and did not consider the possible spatial correlations between individual pavement sections.
Although the earliest record date is in 1947, most record dates are after 1992 (Figure 2(a)). Initial explorations of the data set also show that the PCI records before 1995 may not be fully reliable, since for more than $70 \%$ of the street segments, the PCI values from 1992 to 1994 are exactly the same (see an example of such duplicates in Figure 2(b)). As a result, only data collected in and after 1995 are used for further analysis.

Ageing is a major factor that leads to the degradation of pavement conditions (Paterson, 1987). In the San Francisco PCI data set, only curb-to-curb maintenance projects are recorded. Based on these maintenance records, pavement 'age' since last maintenance is calculated and used as an explanatory variable in degradation models. As only maintenance records can help determine the age of the pavements, PCI records without clear previous maintenance dates are thus removed. A scatter plot of pavement segments' PCI against age based on the whole data set at this stage is given by Figure 3. As there are overlapping data points, all data points are made semi-transparent. Thus, the darker the colour, the more points are located at a position.

Next, some obvious outliers of the PCI records are filtered out. Outliers are defined as survey records with an annual change in PCI larger than 40 (Figure 4(a)). Moreover, as only curb-to-curb maintenance works are documented, it means that small-scale roadworks, such as patching or pothole filling, are not reflected in

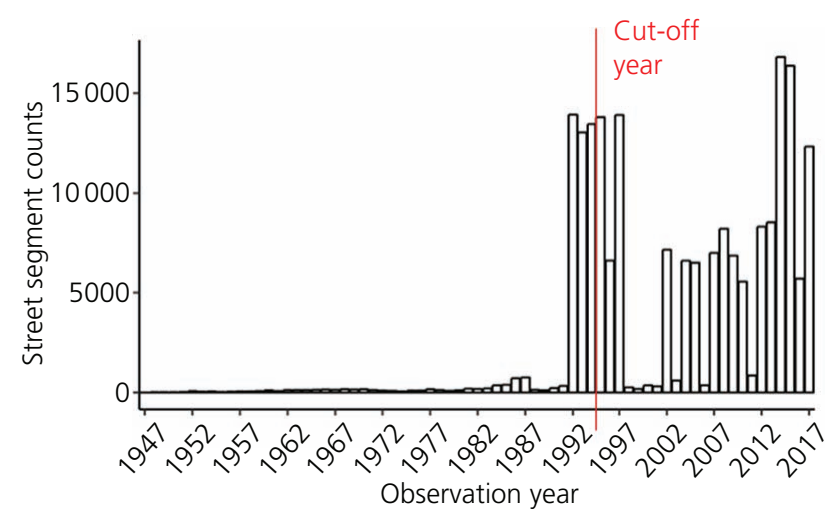

(a)

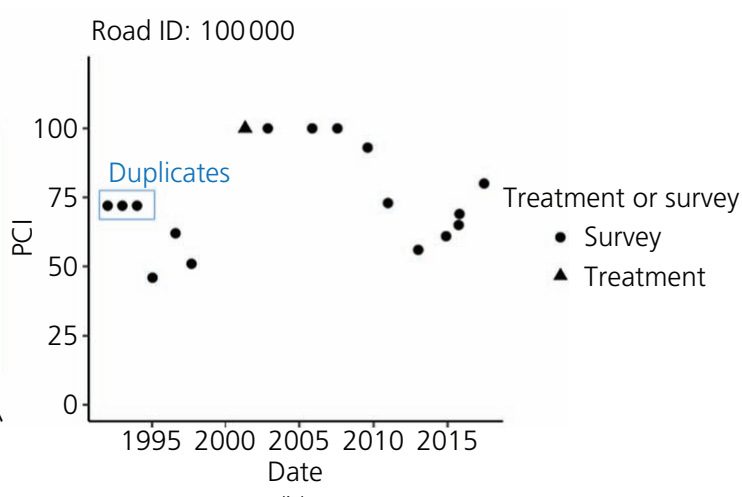

(b)

Figure 2. PCI records: (a) a histogram of the observation dates; (b) an example of duplicated PCI values 1992-1994 
Smart Infrastructure and Construction Volume 171 Issue SC3
Pavement degradation: a city-scale model

for San Francisco, USA

Zhao, Silva and Soga

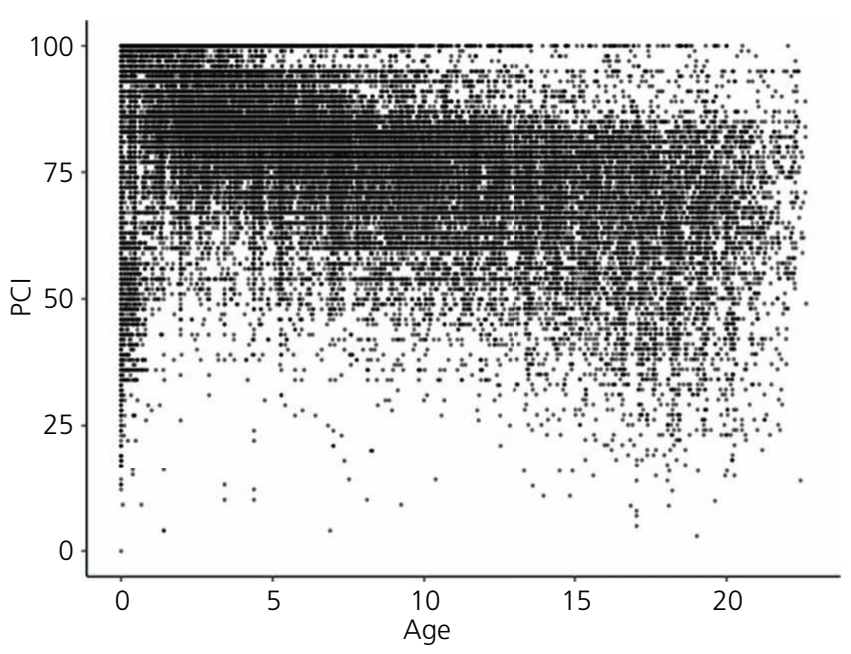

Figure 3. A scatter plot of pavement section's PCI against age

the data set. To mitigate the influence of these missing maintenance records on inferring the degradation rates, streets that show significant improvements (PCI larger than 20 per year) without maintenance have their conditions shifted back to their previous values (Figure 4(b)). This allows the examination of the general degradation trend of a particular road segment between major maintenance events. Survey errors and the absence of some maintenance records are two major limitations in the data. These are two pervasive issues in pavement condition databases and need to be solved - for example, through automated pavement condition surveys or better documentation of roadworks - for better degradation analysis and pavement asset management.

Traffic, material, climate and construction quality also play important roles in the pavement degradation process (Ferreira et al., 2011; Morosiuk et al., 2004). However, not all of these data are well documented or easily accessible. In this study, only the pavement material types and road functional classes are available. Table 2 summarises the number of street segments in each material and functional class category. It can be seen that the majority of streets are asphalt concrete overlaid on top of Portland cement concrete. These categorical characters will be taken into account in the subsequent degradation modelling.

After the above basic data cleaning and processing, 8218 street segments and 49542 PCI records remain in the data set. This equals about five observations per street segment during the study period from 1995 to 2017 (Figure 5). Figure 6 shows the PCI value against pavement age of the cleaned data set, grouped by pavement category. As in Table 2, the majority of the pavements have the surface type ' $C$ ' (asphalt concrete overlaid on top of Portland cement concrete), while few segments have the surface type ' $\mathrm{O}$ ' (asphalt concrete overlaid on top of asphalt concrete). Also from Figure 6, it can be seen that there is a significant variability in pavement degradation trends even for streets belonging to the same category. Simple degradation models based on pavement categories are unlikely to work well in producing accurate predictions on pavement conditions.

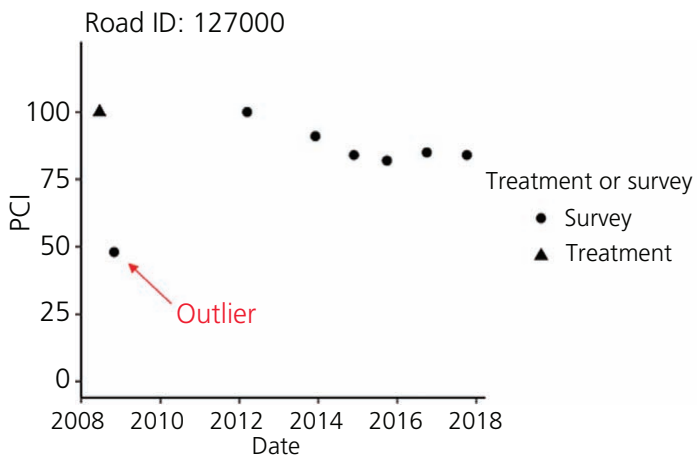

(a)

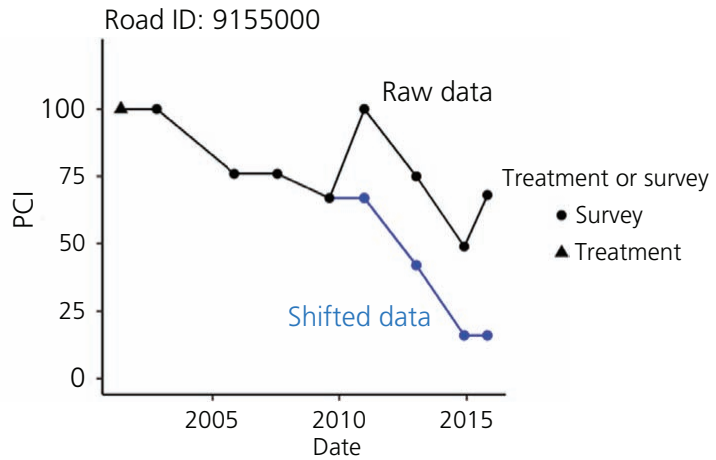

(b)

Figure 4. Data cleaning examples: (a) removing outliers; (b) handling potential missing maintenance records

Table 2. Pavement categories and numbers of street segments in each category (in brackets: abbreviations of category types)

\begin{tabular}{lccc} 
& Arterial (A) & Collector (C) & Residential/local (R) \\
\hline Portland cement concrete (P) & 99 (PA) & 82 (PC) & 1192 (PR) \\
Asphalt concrete overlaid on asphalt concrete (O) & 8 (OA) & NA & 40 (OR) \\
Asphalt concrete overlaid on Portland cement concrete (C) & 13817 (CA) & 3780 (CC) & 30524 (CR)
\end{tabular}




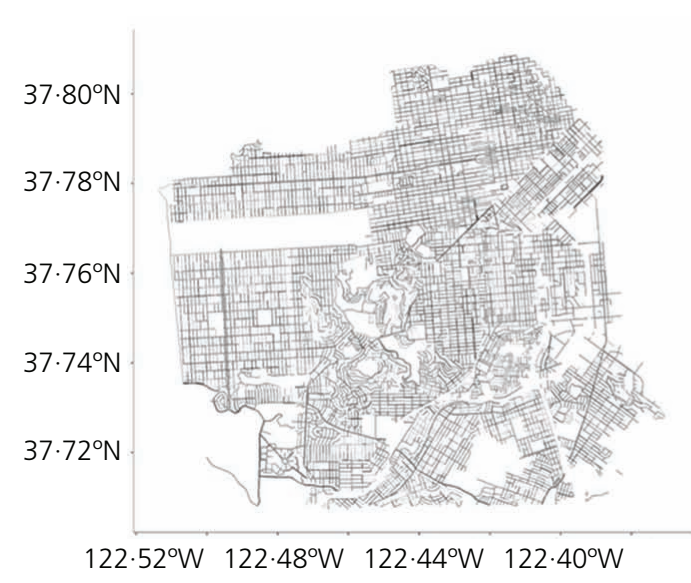

(a)

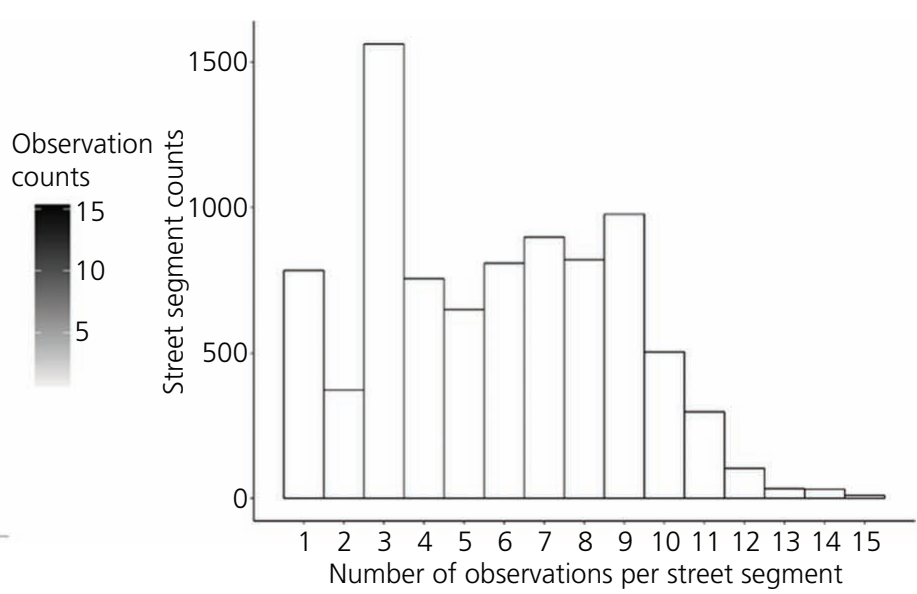

(b)

Figure 5. Numbers of observations per street segment: (a) a spatial view: the darker the street, the more observations available; (b) the histogram

The nearly 50000 pavement condition records from the cleaned data set are randomly split into a training set and a testing set. The training set consists of 39635 records, or roughly $80 \%$ of the whole set. The testing set contains the rest of the 9907 records. In the further analysis, the training set will be used to obtain model coefficients and to evaluate how well the models do in fitting a specific large data set, while the testing set will be used to check the generality - that is, how well the models perform when tested on data unseen.

\section{Methodology}

With pavement age (continuous), surface type (categorical) and street functional class (categorical) as the explanatory variables, a simple and straightforward way to model pavement degradation is as follows: divide all observations in the training data set into categories based on their surface types and functional classes and then build a regression model for pavement age and condition for each category. However, as there are only eight surface type and functional class combinations (Table 2), the categorisation used in this simple model may not be sufficient to represent the diverse pavement characteristics in reality. It is also possible to test the other extreme by considering each street itself as a category and grouping the observed data by street IDs. This allows the individual characters of each street (e.g. climate, geology, construction quality and traffic load) to be captured and represented fully. However, the number of parameters involved in this model will also be large and may lead to overfitting. A third alternative is to choose a medium cluster size, smaller than a street type/functional class category, but larger than an individual street. Spatial modelling offers this capability by incorporating dependencies between neighbouring spatial units and is chosen as the third modelling strategy in this paper.

\subsection{The spatial model}

Spatial models allow smoothly varying coefficients across the entire study area. A simple way to incorporate spatial relationships in a model is to include the longitude and latitude coordinates as model predictors and fit a trend surface. However, such models can only capture global trends if not using high-order terms (Lichstein et al., 2002). To represent localised interactions, model structures such as geographically weighted regression, simultaneous autoregression (SAR) and conditional autoregression are more suitable. These model structures consider spatial dependencies between neighbours by imposing constraints on the values (or residuals) of neighbouring sites (Dormann et al., 2007). Readers can refer to the report of Jahanbakhsh et al. (2016) for an SAR model that predicts pavement conditions with spatial and temporal lags. This study is designed to model street-specific degradation rates as the average of neighbouring rates, so an intrinsic conditional autoregressive 'Besag' model becomes a natural choice (Besag et al., 1991; Blangiardo et al., 2013; Lavine and Hodges, 2012).

Pavement degradation with spatial effects can be formulated as the following multilevel model (Blangiardo and Cameletti, 2015; Rue et al., 2009): the first step is to model the distribution of the observations

$$
\text { 1. } y_{i k} \sim N\left(\eta_{i k}, \frac{1}{\tau_{g}}\right)
$$

where $y_{i k}$ is the $k$ th observed PCI at street $i$, given that most of the street segments have more than one record in the past 20 years (Figure 5). $y_{i k}$ is assumed to follow a normal distribution with mean $\eta_{i k}$ and precision $\tau_{g}$ (inverse of variance). The distribution equation states that the observed PCI centres around an unobservable mean $\eta_{i k}$, plus some random deviations determined by the precision parameter $\tau_{g}$. In the second step, $\eta_{i k}$ is modelled by a linear combination of the explanatory variables

$$
\text { 2. } \eta_{i k}=\alpha+\xi_{i}+\left(\beta+v_{i}+u_{i}\right) x_{i k}
$$




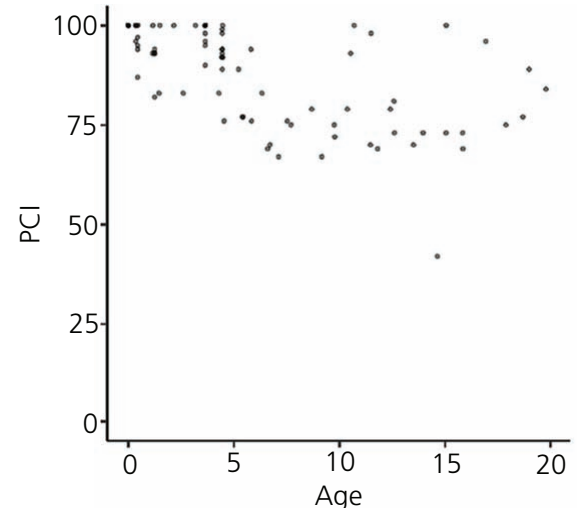

(a)

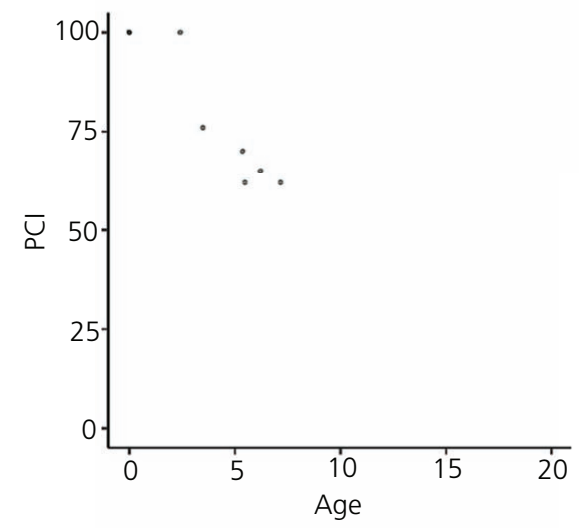

(d)

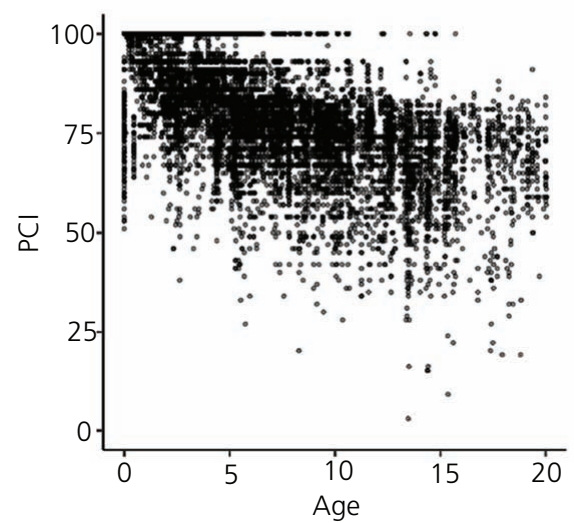

(g)

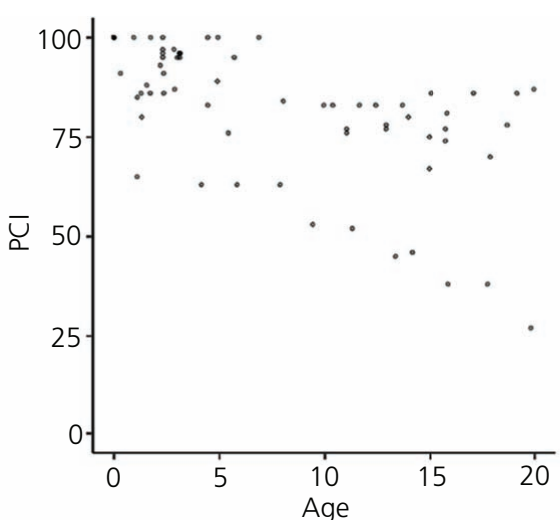

(b)

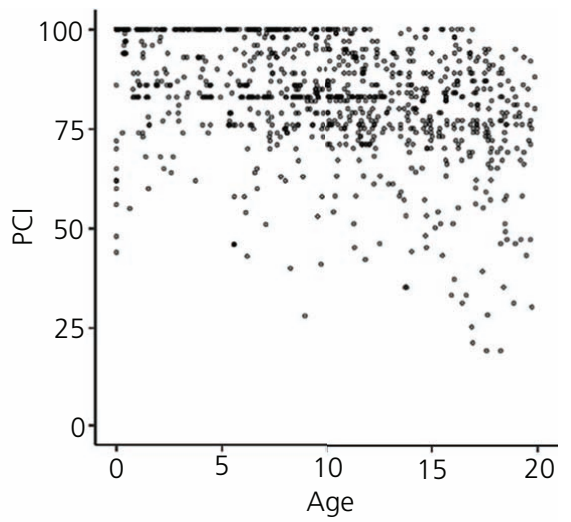

(c)

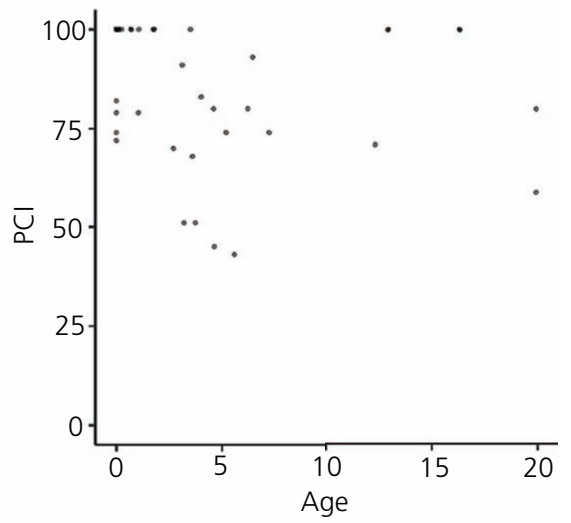

(e)

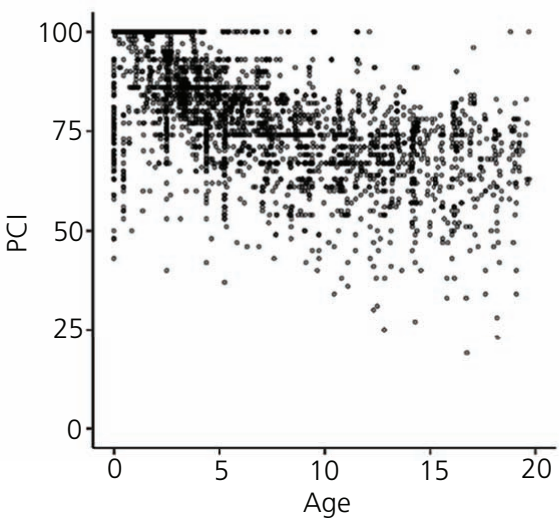

(h)

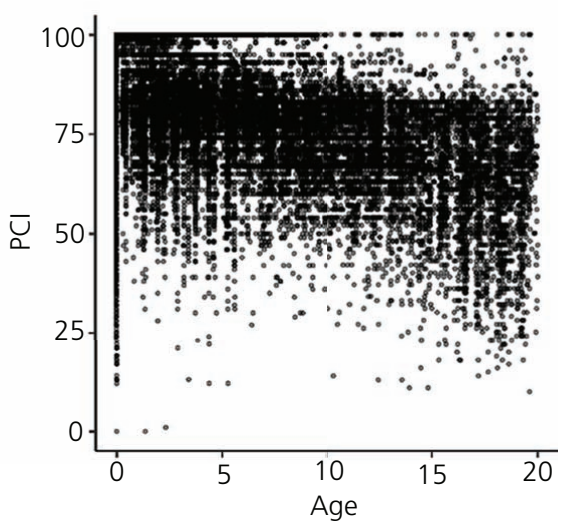

(i)

Figure 6. PCl plotted against pavement age, grouped by pavement category: (a) PA; (b) PC; (c) PR; (d) OA; (e) OC; (f) OR; (g) CA; (h) CC; (i) CR. Refer to Table 2 for meanings of abbreviations

3. $\xi_{i} \sim N\left(0, \frac{1}{\tau_{\xi}}\right)$

4. $u_{i} \sim N\left(0, \frac{1}{\tau_{u}}\right)$
5. $v_{i} \mid v_{j \neq i}, \tau_{v} \sim N\left(\frac{1}{n_{i}} \sum_{j \sim i} v_{j}, \frac{1}{n_{i} \tau_{v}}\right)$

$x_{i k}$ is the explanatory variable (age) corresponding to observation $y_{i k} . \alpha$ and $\beta$ are the global average intercept and age effect shared by all streets. $\xi_{i}$ is the street-specific variation in intercept, which 
Smart Infrastructure and Construction

Volume 171 Issue SC3
Pavement degradation: a city-scale model

for San Francisco, USA

Zhao, Silva and Soga itself is a random variable following a zero-mean normal distribution (Equation 3). $\beta+v_{i}+u_{i}$ is the total street-specific age effect for street $i$, where $v_{i}$ is the spatially structured individual deviation from the mean and $u_{i}$ is the unstructured part. A Besag specification is adopted for modelling $v_{i}$ (Besag et al., 1991; Blangiardo et al., 2013): as shown in Equation 5, $v_{i}$ is a Gaussian random variable whose mean equals the average of neighbouring sites' values $v_{j}(j \sim i$ means $i$ and $j$ are neighbours) and whose precision $\tau_{v}$ is to be estimated from the data. $n_{i}$ is the number of neighbours that street $i$ has. Since $v_{i}$ is related only to its neighbours, $v_{i}$ and $v_{l}$ are conditionally independent if $i$ and $l$ are not neighbours (the Markov property). So $\boldsymbol{v}=\left\{v_{1}, v_{2}, \ldots\right\}$ is said to be a Gaussian Markov random field.

In the third step of the multilevel model, prior distributions are assigned to model parameters. Details on prior distributions will be given in Section 6. A graphical model for the three-level spatial model is given in Figure 7(d).

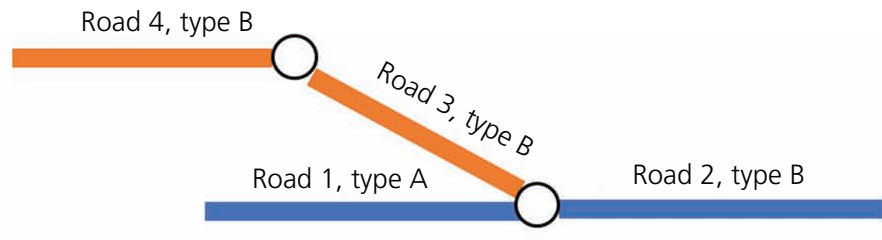

(a)
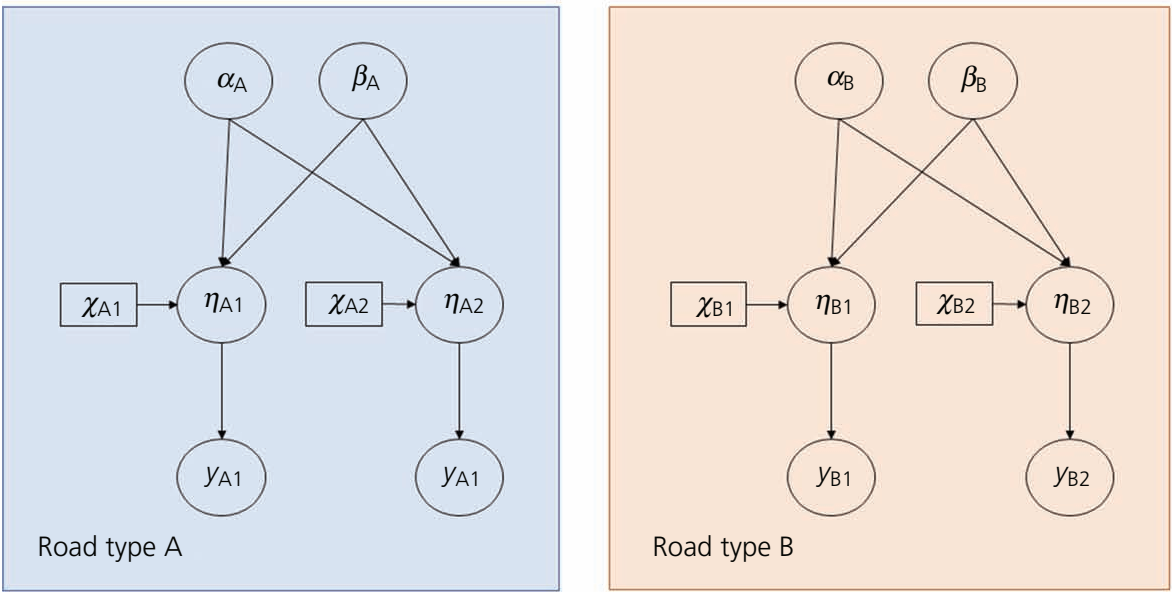

(b)
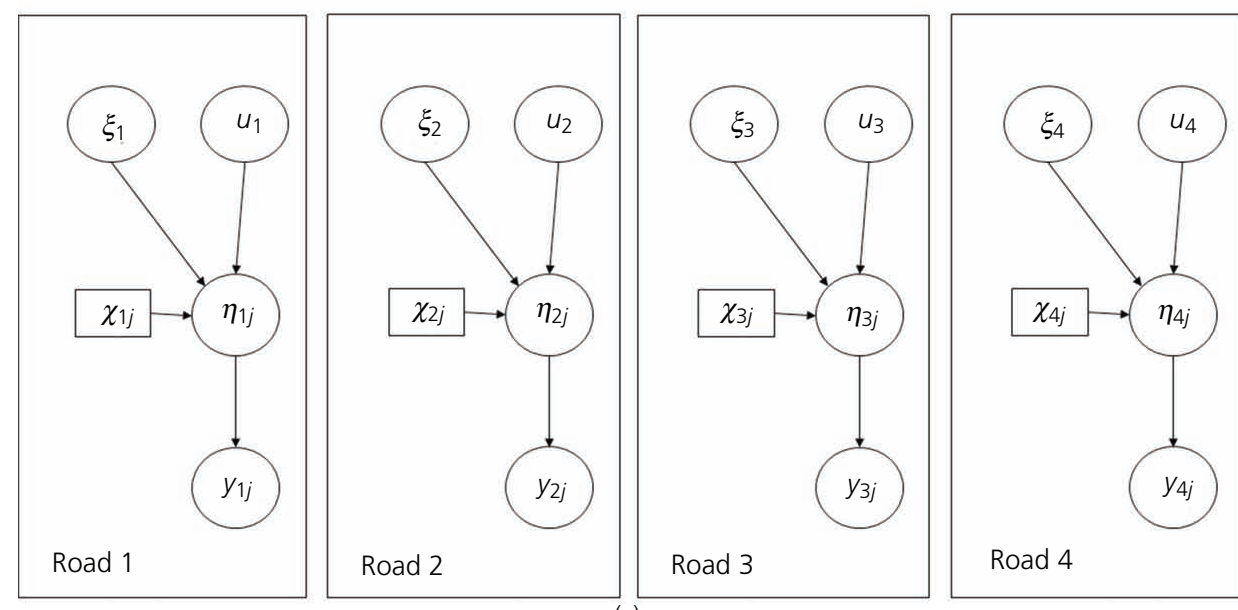

(c)

Figure 7. Graphical models of road degradation. Symbols are consistent with definitions in Equations 1-5 and Table 3. For clarity, random noises are not shown in the graphical models. Global-level variables ( $\alpha$ and $\beta$ in models NSP- 2 and SP, as well as the hyperparameters) are not shown either. (a) An example network made of four road segments and two road type categories; (b) NSP-1: coarse categorisation based on road type category; (c) NSP-2: fine categorisation based on individual roads; (d) SP: spatial models with correlated parameters between neighbouring road segments (continued on next page) 


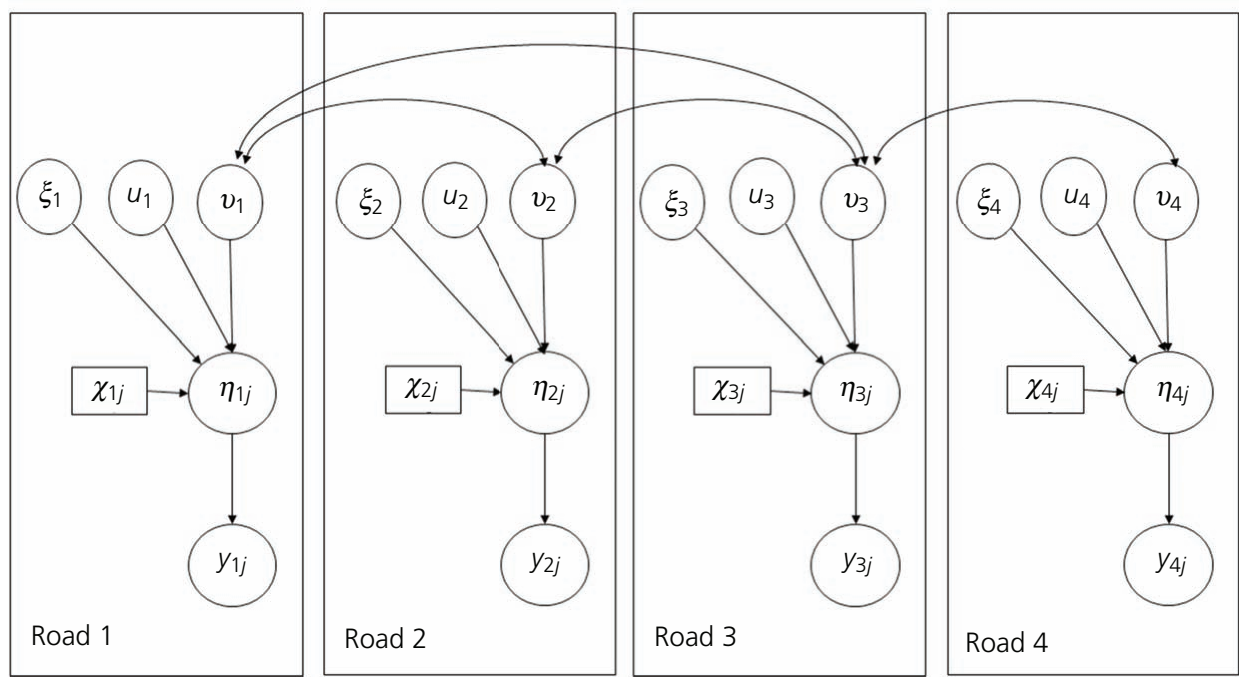

(d)

Figure 7. Continued

\subsection{Bayesian regression using R-INLA}

For multilevel models such as the one presented earlier, various techniques are available for parameter inference, including maximum likelihood estimation and the Markov chain Monte Carlo (MCMC) method (Croissant and Millo, 2008; Gelman and Hill, 2006). Rue et al. (2009) demonstrated a direct-approximationbased Bayesian approach, called INLA, to be fast and sufficiently accurate for parameter inferences of spatial hierarchical models. In the preliminary stage of this study, the MCMC approach also showed promising and comparable results. Since the scope of this paper is to compare spatial and non-spatial model structures rather than the various inference methods, only INLA, the most flexible approach according to the authors' experience, is adopted.

Continuing with the notation definitions in Equations $1-5$, the task of regression is to estimate model parameters $\boldsymbol{\theta}=\{\alpha, \beta, \boldsymbol{\xi}, \boldsymbol{v}$, $\boldsymbol{u}\}$ and hyperparameters $\boldsymbol{\psi}=\left\{\tau_{g}, \tau_{x i}, \tau_{u}, \tau_{v}\right\}$ from the data. Based on the Bayes theorem and conditional probability, the joint posterior distribution $\pi(\boldsymbol{\theta}, \boldsymbol{\psi} \mid \boldsymbol{y})$ is given by

6. $\pi(\boldsymbol{\theta}, \boldsymbol{\psi} \mid \boldsymbol{y}) \propto \pi(\boldsymbol{y} \mid \boldsymbol{\theta}, \boldsymbol{\psi}) \pi(\boldsymbol{\theta} \mid \psi) \pi(\psi)$

From Equation 6, marginal posteriors of a parameter, $p\left(\theta_{w} \mid \boldsymbol{y}\right)$, and a hyperparameter, $p\left(\psi_{h} \mid \boldsymbol{y}\right)$, can be obtained through integration

7.

$$
\pi\left(\theta_{w} \mid \boldsymbol{y}\right)=\int \pi\left(\theta_{w} \mid \psi, y\right) \pi(\psi \mid y) d \psi
$$

$\pi\left(\psi_{h} \mid \boldsymbol{y}\right)=\int \pi(\boldsymbol{\psi} \mid \boldsymbol{y}) d \psi_{-h}$
INLA does the preceding integrations through approximating the integrands with known distributions. Based on the paper of Tierney and Kadane (1986), Rue and Martino (2007) proposed the following approximation

$$
\text { 9. } \pi(\psi \mid y)=\left.\frac{\pi(\boldsymbol{\theta}, \psi \mid y)}{\pi(\boldsymbol{\theta} \mid \psi, y)} \propto \frac{\pi(\boldsymbol{\theta}, \psi, y)}{\widetilde{\pi}_{\mathrm{G}}(\boldsymbol{\theta} \mid \psi, y)}\right|_{\boldsymbol{\theta}=\boldsymbol{\theta}^{*}{ }_{(\psi)}}
$$

where $\tilde{\pi}_{\mathrm{G}}(\boldsymbol{\theta} \mid \boldsymbol{\psi}, \boldsymbol{y})$ is the Gaussian approximation of $\pi(\boldsymbol{\theta} \mid \boldsymbol{\psi}, \boldsymbol{y})$ near its mode $\theta^{*}(\psi)$. The formula is equivalent to the Laplace approximation of marginal posterior density in the paper of Tierney and Kadane (1986). Similarly, the other integrand in Equation 7 is approximated by (Rue et al., 2009)

10.

$$
\begin{aligned}
\pi\left(\theta_{w} \mid \psi, y\right) & =\frac{\pi\left(\theta_{w}, \boldsymbol{\theta}_{-w} \mid \psi, y\right)}{\pi\left(\boldsymbol{\theta}_{-w} \mid \theta_{w}, \psi, \boldsymbol{y}\right)} \\
& \left.\propto \frac{\pi(\boldsymbol{\theta}, \boldsymbol{\psi}, \boldsymbol{y})}{\widetilde{\pi}_{\mathrm{G}}\left(\boldsymbol{\theta}_{-w} \mid \theta_{w}, \psi, \boldsymbol{y}\right)}\right|_{\boldsymbol{\theta}_{-w}=\boldsymbol{\theta}_{-w}^{*}(\boldsymbol{\psi})}
\end{aligned}
$$

Substituting the integrands in Equations 7 and 8 with Equations 9 and 10 , the marginal posterior distributions become integrations at a much lower dimension, which can then be solved numerically. In this study, the R package 'INLA' (R-INLA Project, 2019) is used for the Bayesian INLA regression. Apart from the methodological references by Rue and Martino (2007) and Rue et al. (2009), information about INLA applications can also be found in the publications by Blangiardo et al. (2013) and Schrödle and Held (2011).

\section{Models}

As discussed in Section 4, three pavement degradation models are designed to represent an array of modelling strategies 
NSP-1: a non-spatial model with data divided into coarse categories based on pavement surface type and street functional class

- NSP-2: a non-spatial model with data divided into fine categories based on street ID

v SP: a spatial model with fine categories (i.e. fine spatial units based on street ID).

The mathematical forms for these models are given in Table 3 . Directed acyclic graphic (DAG) models showing variable relationships are provided in Figure 7. For clarity, the DAG models in Figure 7 are based on a simplified network which consists of only four road segments in two pavement categories (Figure 7(a)), as opposed to the 8218 segments and eight pavement categories (Table 2) in the real data set. Nonetheless, the simplified network and DAG models in Figure 7 are sufficient to illustrate the structures and differences of the three models.

\subsection{NSP-1}

NSP-1 is a simple model where pavement condition observations are divided into categories first based on the surface materials and street functional classes, as shown in Figure 6. Separately in each category, the condition degradation is modelled with a non-spatial linear form, where age is the main explanatory variable. Visually, this is equivalent to fit a linear trend to data in each cell in Figure 6. Although more complex model forms can be used, such as the non-linear trend used by the California MTC (Deshmukh, 2010), a linear form is a good starting point for showing the overall trend and comparing with models NSP-2 and SP, which are also linear in nature.

This model is represented by the DAG in Figure 7(b). $y_{\mathrm{A} 1}$ and $y_{\mathrm{A} 2}$ are PCI observations of pavements belonging to category A. $x_{\mathrm{A} 1}$ and $x_{\mathrm{A} 2}$ are corresponding pavement ages (the explanatory variable). $\quad \alpha_{\mathrm{A}}$ and $\beta_{\mathrm{A}}$ are the intercept and age effect for pavements belonging to category A. For the linear model specified by NSP-1, $\eta_{\mathrm{A} 1}$, the unobserved mean of $y_{\mathrm{A} 1}$, is calculated as the linear combination of all the incoming nodes: $\alpha_{\mathrm{A}}+\beta_{\mathrm{A}} * x_{\mathrm{A} 1}$ and $\eta_{\mathrm{A} 2}=\alpha_{\mathrm{A}}+\beta_{\mathrm{A}} * x_{\mathrm{A} 2} . \alpha_{\mathrm{A}}$ and $\beta_{\mathrm{A}}$ contribute to all data in category $\mathrm{A}$. The same applies to data in category $\mathrm{B}$, except that they use a separate set of parameters, $\alpha_{\mathrm{B}}$ and $\beta_{\mathrm{B}}$. As a result, data and variables for category $\mathrm{A}$ and category $\mathrm{B}$ are put into separate boxes and no link exists between them.

\subsection{NSP-2}

As each street segment may have its own characteristics, model NSP-2 refines the categories in NSP-1 by treating each street as a category itself - for example, a separate box for each road segment, as shown in Figure 7(c). A linear trend is fitted for each street segment. $\alpha$ and $\beta$ are the global average intercept and age coefficient, and these two variables are shared by data in all categories (not shown but contributing to all $\eta_{i j}$ values in Figure 7(c)). Moreover, for each street segment $i, \xi_{i}$ and $u_{i}$ are the individual street's deviations in intercept and age effect from the global mean. Both $\xi_{i}$ and $u_{i}$ are assumed to be independent and identically distributed variables (normally distributed, to be specific), and they contribute to $\eta_{i j}$ only within the same box in Figure 7 (c).

The differences between model NSP-1 and NSP-2 not only lie in the numbers of the categories. Furthermore, the intercepts and age effects in model NSP-1 are allowed to vary without constraints, while these parameters in NSP-2 have to satisfy global normal distribution constraints, controlled by precision variables $\tau_{u}$ and $\tau_{\xi}$. This is because, as the categories become finer, fewer data points exist for each category and regression coefficients will be significantly affected by errors in the data points. To prevent

Table 3. Models

\begin{tabular}{|c|c|c|c|}
\hline Number & Features & Model & Definitions \\
\hline NSP-1 & Global level; regression by category & $y_{l k}=\alpha_{l}+\beta_{l} x_{l k}$ & $\begin{array}{l}\text { I: index for road type and functional class category } \\
y_{l k}: k \text { th observation in category I } \\
x_{l k}: \text { corresponding pavement age for } y_{l k} \\
\alpha_{l} \text { and } \beta_{l} \text { : regression parameters for category I }\end{array}$ \\
\hline NSP-2 & $\begin{array}{l}\text { Street level; spatially unstructured } \\
\text { intercepts and age effects }\end{array}$ & $\begin{array}{l}y_{i k}=\alpha+\xi_{i}+\left(\beta+u_{i}\right) x_{i k} \\
\xi_{i} \sim N\left(0, \tau_{\xi}^{-1}\right) \\
u_{i} \sim N\left(0, \tau_{u}^{-1}\right)\end{array}$ & $\begin{array}{l}i: \text { index for road segment } \\
y_{i k}: k \text { th observation for road } i \\
x_{i k}: \text { corresponding pavement age for } y_{i k} \\
\alpha \text { and } \beta \text { : global average intercept and age effect } \\
\xi_{i}: \text { street-level intercept deviation from } \alpha \\
u_{i} \text { : street-level age effect deviation from } \beta \\
\tau_{\xi}: \text { precision for the independent and identically distributed } \\
\text { (iid) variable } \xi \\
\tau_{u}: \text { precision for the iid variable } u\end{array}$ \\
\hline SP & $\begin{array}{l}\text { Street level; spatially unstructured } \\
\text { intercepts and spatially structured } \\
\text { age effects }\end{array}$ & $\begin{array}{l}y_{i k}=\alpha+\xi_{i}+\left(\beta+u_{i}+v_{i}\right) x_{i k} \\
\xi_{i} \sim N\left(0, \tau_{\xi}^{-1}\right) \\
u_{i} \sim N\left(0, \tau_{u}^{-1}\right) \\
v_{i} \mid v_{j, i \neq j}, \tau_{v} \sim N\left(\frac{1}{n_{i}} \sum_{i-j} v_{j}, \frac{1}{n_{i} \tau_{v}}\right)\end{array}$ & $\begin{array}{l}i, y_{i k}, x_{i k}, \alpha, \beta, \xi_{i}, \tau_{\xi}, \tau_{u}: \text { same as in NSP-2 } \\
\text { ui: the spatially unstructured street-level age effect } \\
\text { deviation from } \beta \\
v_{i} \text { : the spatially structured street-level age effect deviation } \\
\text { from } \beta \\
i \sim j: i \text { and } j \text { are neighbours } \\
\tau_{v} \text { : the-precision related parameter for spatially structured } \\
\text { random variable } v\end{array}$ \\
\hline
\end{tabular}


unrealistically large or small intercepts and age effects from being obtained, global constraints are thus imposed to limit their variation ranges. It will be shown in the coming section that the spatial model addresses the same issue, but with a spatially correlated parameter.

\subsection{SP}

As there are more than 8000 street segments in the cleaned data set, model NSP-2 also includes thousands of parameters. However, most of the additional parameters $\left(\xi_{i}\right.$ and $\left.u_{i}\right)$ are random effects that serve to improve the model fit without revealing specific reasons or patterns. In comparison, model SP partitions the street-level age effect into two parts: the spatially structured $v_{i}$ and spatially unstructured $u_{i}$. This is shown graphically in Figure 7(d), where $\eta_{i j}$, now having six parent nodes ( $\alpha$ and $\beta$ not shown for clarity of the figure), is calculated as $\alpha+\xi_{i}+\left(\beta+u_{i}+v_{i}\right) * x_{i j}$.

The spatially structured $v_{i}$ varies smoothly across the space and makes clusters of road segments based on their degradation rates. Specifically, $v_{i}$ is assumed to follow the Besag specification (Equation 5), with mean value equal to the average of the spatial components of its neighbours. This is the unique feature and an advantage of the spatial model, which is to borrow information/ strength from neighbours'. Neighbours are defined as adjacent road segments. The more neighbours that street segment $i$ has, the smaller the variance of $v_{i}$. Similar to NSP-2, the spatially unstructured random effects in SP $\left(\xi_{i}\right.$ and $\left.u_{i}\right)$ are also subjected to the global distribution constraints. The spatially structured $v_{i}$, on the contrary, can be viewed as localised constraints on the parameter values.

\section{Results}

Parameter estimations for the degradation models are carried out in software R 3.4.1 (R Core Team, 2017). Priors are specified as follows: for $\alpha$, the PCI of newly constructed pavement, its physical meaning limits its value to be close to 100. A normal prior with large variance $N\left(0,0 \cdot 0001^{-1}\right)$ is adopted for $\alpha$. With sufficient data, it is expected that the prior is only weakly informative, and the posterior distribution will be much narrower than the prior. The same rationale applies to $\beta$, the PCI degradation rate, whose actual value is estimated to be a small negative number around -5 to 0 , and $N\left(0,0.0001^{-1}\right)$ is again chosen as the prior. The hyperparameters $\tau_{\xi}, \tau_{u}, \tau_{v}$ and $\tau_{g}$ are 'precision' parameters that are mathematically constrained to be positive. So the Gamma distribution with a large variance, Gamma(1, 0.0005), is used as their priors. The Gamma distribution is parameterised with the shape and rate parameters, while the normal distribution is parameterised with mean and variance (inverse of precision).

Resulting street-level age-zero condition and degradation rate under these specified priors are shown in Figure 8 as maps. Moreover, Table 4 provides detailed numerical summaries of the results, including $(a)$ values or distributional characters of the regression coefficients $\alpha, \beta, \boldsymbol{x i}, \boldsymbol{u}$ and $\boldsymbol{v} ;(b)$ the rootmean-square errors (RMSEs) on the training and the testing data sets; and (c) the DIC for comparing Bayesian models (Spiegelhalter et al., 2002), which will be explained in detail later in this section.

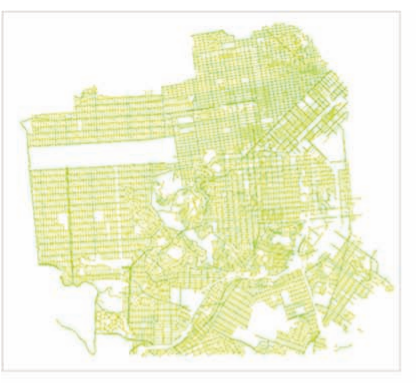

(a)

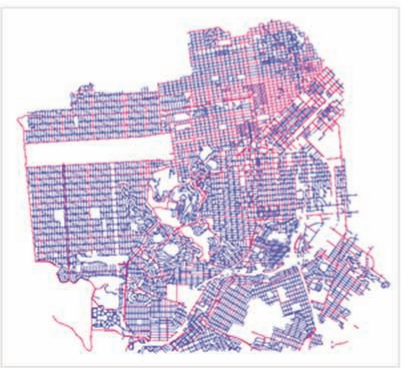

(d)

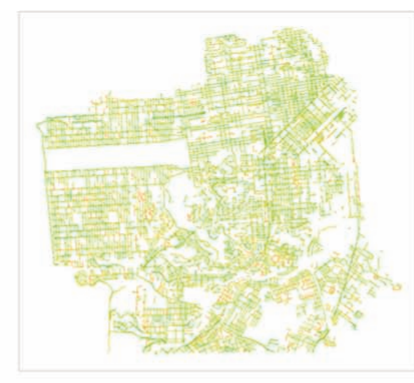

(b)

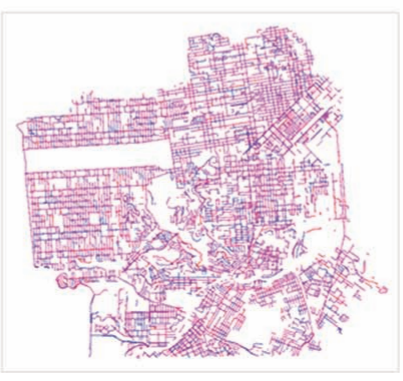

(e)

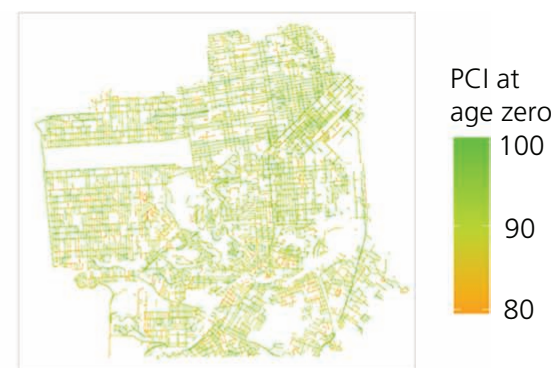

(c)

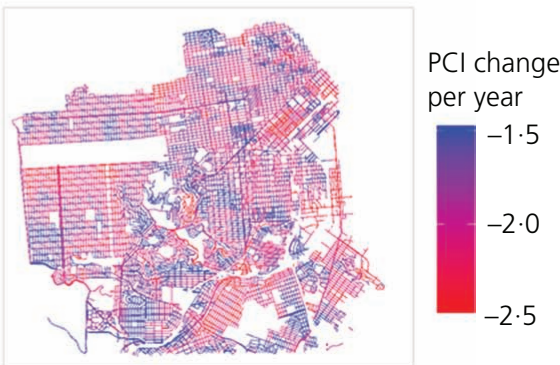

$(f)$

Figure 8. Map view of regression coefficient results. (a) NSP-1: $\alpha$, (b) NSP-2: $\alpha+\xi_{i}$ (c) SP: $\alpha+\xi_{i}$ (d) NSP-1: $\beta ;$ (e) NSP-2: $\beta+u_{i}$ (f) SP: $\beta+$ $u_{i}+v_{i}$ 


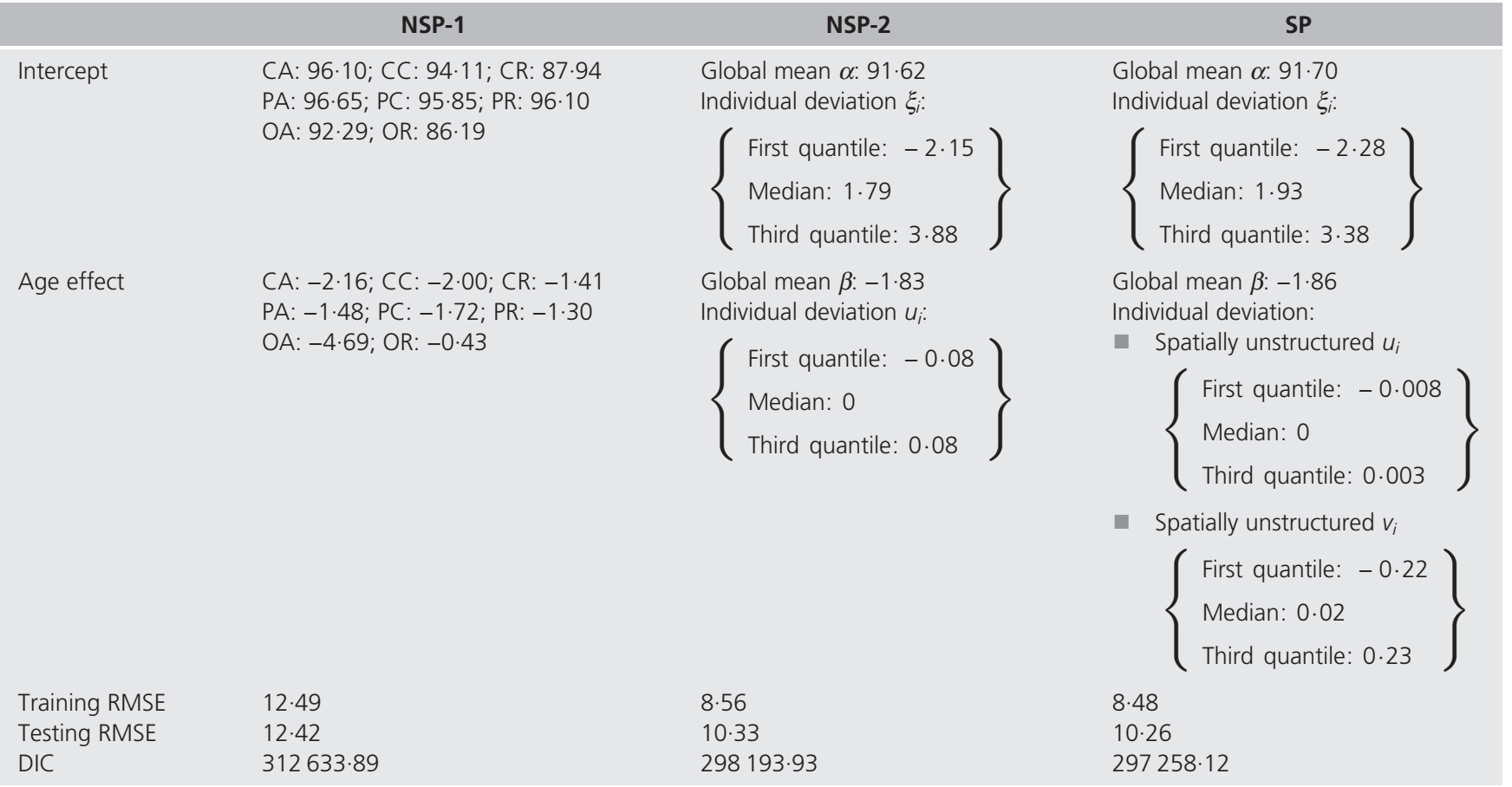

DIC, deviance information criterion; RMSE, root-mean-square error

Prior specifications in INLA may have significant impacts on regression results. Also, priors affect model complexity: the wider the prior is, the more complex (and undesirable) the model becomes. To test whether the results and conclusions still hold under different priors, a sensitivity analysis is carried out and presented at the end of this section.

\subsection{NSP-1}

In NSP-1, regression coefficients ( $\alpha$ and $\beta$ ) are obtained based on street type category and the resulting degradation trends are plotted alongside the data in Figure 9. Residential roads (type '*R', the rightmost column in Figure 9) are found to have the lowest degradation rates across all surface types, probably due to the less heavy traffic they carry. Streets with asphalt overlaid surface (types 'OA' and 'OR', Figures 9(d) and 9(f)) have the most extreme degradation rates: the yearly PCI change for OA roads is as fast as -4.69 , while for OR roads, it is as slow as $-0 \cdot 43$. This may be the consequence of the small numbers of observations for these two types of roads in the data set.

Additionally, NSP-1 results are presented in different forms as in Figures 8(a) and 8(d), Table 4 (under the column 'NSP-1') and Figures 10(a) and 10(b) (leftmost clusters). They will be compared and discussed with the other two models.

\subsection{NSP-2}

Model NSP-2 fits a linear trend for each street segment. Results suggest that, on the average, the initial PCI condition is 91.62 and drops by 1.83 every year after. The street-level initial conditions and degradation rates are shown as maps in Figures 8(b) and 8(e).

Additionally, Figure 10 is provided for more visual and detailed comparison of the results. In this figure, each point stands for the regression coefficient for a group or a street segment. The points are clustered and coloured based on street types. Black horizontal bars mark the locations of the tenth, 20th, ..., 90th percentiles for each group. Comparing NSP-2 results with those from NSP-1, it can be seen that the regression coefficients are scattered even within the same street category. For example, in NSP-2, the 30th percentile street-level degradation rates of Portland cement concrete, residential streets (type 'PR') is $-1 \cdot 33$, while the 70 th percentile degradation rate of the same type of street is $-1 \cdot 80$, almost 35\% faster. Simple group-level results as in NSP-1 cannot adequately capture the individual variations in degradation trends.

\subsection{SP}

Model SP is a spatial extension of NSP-2 that partitions the streetspecific degradation rate into two parts: the spatially structured $v_{i}$ and the spatially unstructured $u_{i}$. Maps showing the resulting street-level coefficients from model SP are given by Figures 8(c) and $8(\mathrm{f})$. The resulting street-level initial condition map is similar to that of NSP-2, possibly because the intercepts are treated as spatially unstructured in both models. However, maps of the degradation rates are considerably different: in NSP-2, degradation rates are estimated largely based on individual road segments. In comparison, in SP, the regressions are coordinated between several street segments within the defined neighbourhood. The 


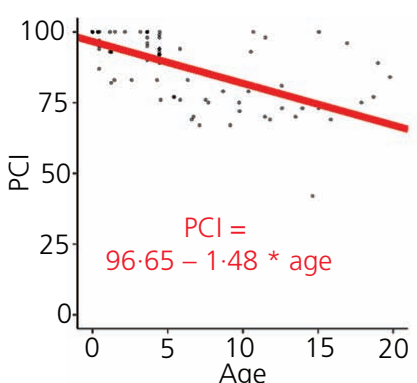

(a)

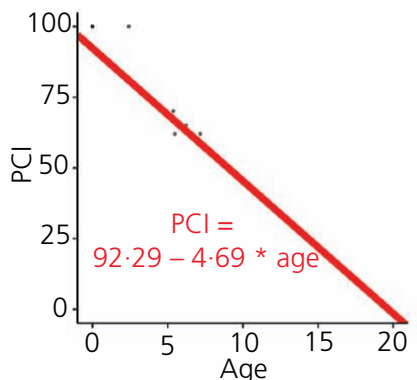

(d)

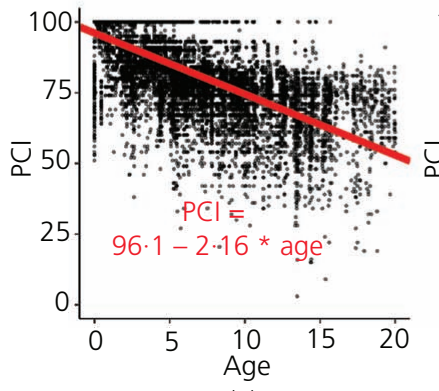

(g)

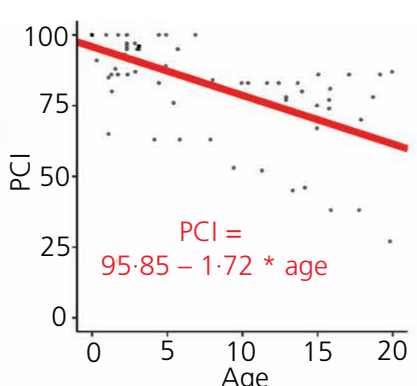

(b)

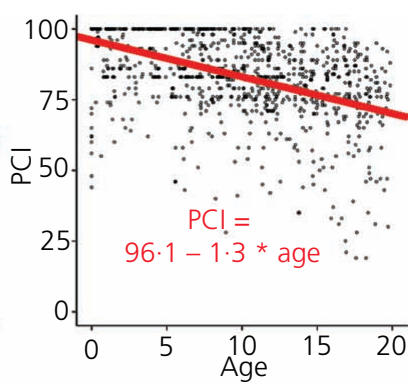

(c)

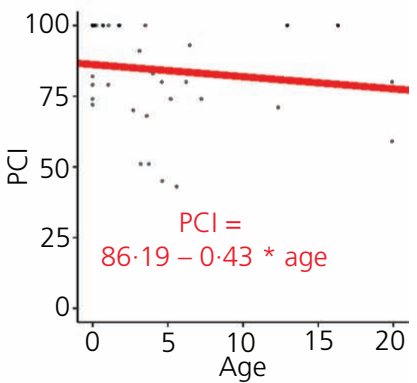

(f)

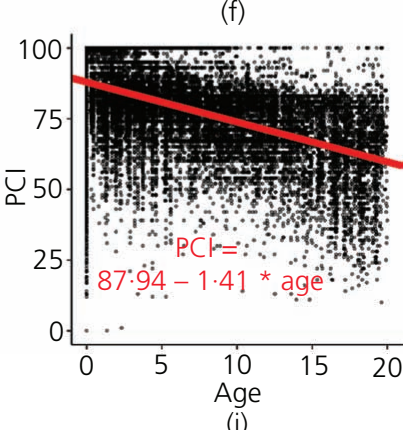

(h)

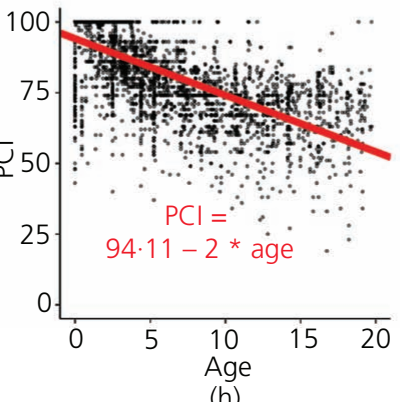

Figure 9. Regression results from model NSP-1: (a) PA; (b) PC; (c) PR; (d) OA; (e) OC; (f) OR; (g) CA; (h) CC; (i) CR. See Table 2 for the meanings of abbreviations

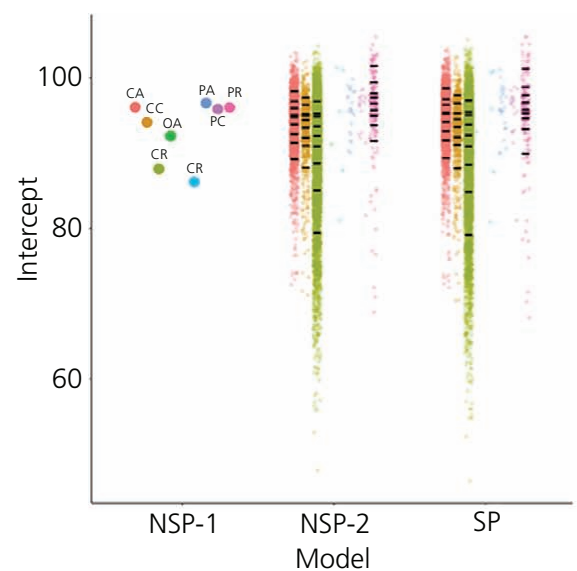

(a)

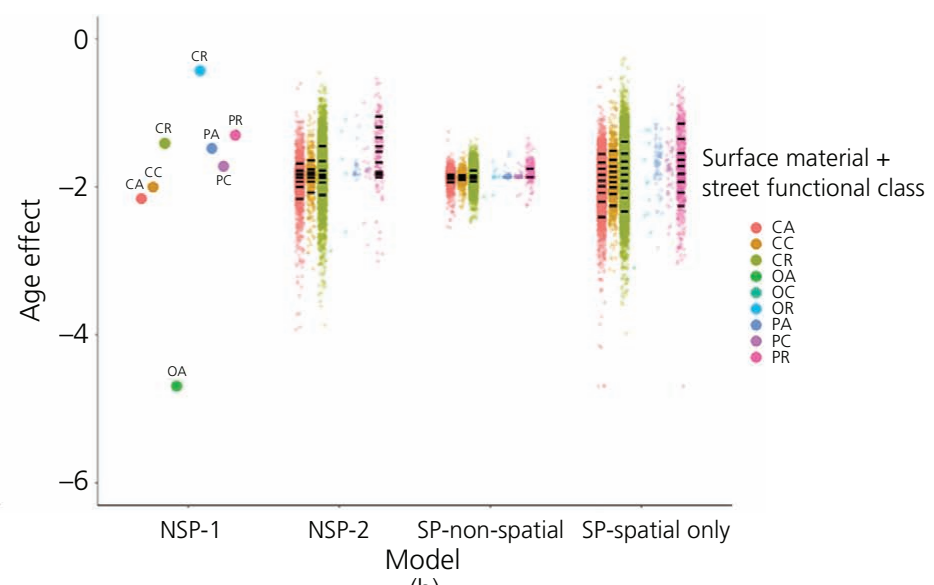

(b)

Figure 10. Resulting intercepts and age effects from different models: (a) intercepts; (b) age effects. See Table 2 for meanings of abbreviations 
degradation rate map from model NSP-2 does not exhibit any special structure or pattern (Figure 8(e)), while for model SP, it shows regions of high (in red) and low degradation rates (in blue) (Figure 8(f)). This spatial pattern can be further studied to reveal underlying causes of the differences in degradation rates.

To compare the spread/distributional characters of the regression results from models NSP-2 and SP, Figure 10 is again used. In Figure 10(a), the variations of the intercepts are similar based on model NSP-2 and SP. In Figure 10(b), the resulting degradation rates from model SP are further divided into two parts: 'SP-nonspatial' shows only the non-spatial components (i.e. $\beta+u_{i}$ ), while 'SP-spatial only' shows the spatial components (i.e. $\beta+v_{i}$ ). The variation range of $v_{i}$ is significantly larger than $u_{i}$. In other words, a large part of the individual variations in degradation rates are explained by the spatially structured component.

\subsection{Model comparison using RMSE and DIC}

The last three rows in Table 4 give metrics for model comparison. Among them, the RMSEs of the training and testing data sets are used to compare the fitting and predicting abilities of the models. NSP-1, due to its oversimplified structure, has the worst fitting/ predicting performance. In fact, it underfits the data, as the testing RMSE (12.42) is about the same as the training RMSE (12.49). NSP-2 performs better than NSP-1, with the training RMSE (8.56) and testing RMSE $(10 \cdot 33)$ both being smaller. The RMSE on the testing data set is slightly higher than the training RMSE, but not large enough to be considered as overfitting. Model SP has the smallest training RMSE (8.48) and testing RMSE (10.26) among all three models. Compared with NSP-2, the improvements in fitting and predicting accuracy are not a significant advantage of model SP. The most important strength of model SP is that it has identified regions of high degradation rates. In fact, the spatial component is rather dominant in the total variability of degradation rates based on model SP (Figure 10(b)). If the pavement degradation rates do not possess spatial features, results would be expected that the values of the spatial component $v_{i}$ being closer to zero for all roads. In other words, as the data size for individual pavement is small, estimating its degradation rate is greatly affected by outliers (measurement errors). Model NSP-2 addresses this through identical and independent prior constraints on the parameters, while model SP introduces spatially correlated parameters. The spatial component is found to be dominant, also proving that the spatially correlated constraints are a reasonable problem-specific adaptation of the independent constraints.

DIC is another metric for comparing Bayesian models. It is calculated by using either of the following formulas (Spiegelhalter et al., 2014)

11. $\mathrm{DIC}=D(\bar{\theta})+2 p_{D}$

12. $\mathrm{DIC}=\bar{D}+p_{D}$ where $D$ (deviance) is a goodness-of-fit statistics equalling $2 \log$ (likelihood). A smaller deviance indicates a better fit to the data. $D(\bar{\theta})$ is the deviance at posterior means (a classical pointwise measure of model fit), and $\bar{D}$ is the posterior mean deviance (a Bayesian measure of model fit). $p_{D}=\bar{D}-D(\bar{\theta})$ is the effective number of parameters and reflects the complexity/degree of 'overfitting' of the posterior distributions. $p_{D}$ is included in DIC to penalise complex models. DIC combines the goodness-of-fit measure $(D)$ and the effective number of parameters $\left(p_{D}\right)$, thus reflecting both model fit and complexity. A smaller DIC is better as it indicates a more desirable balance of fit and complexity. The absolute value of DIC is not meaningful, and models are compared based on their differences in DIC. From Table 4, SP has the smallest DIC and is the preferred choice among the three models.

\subsection{Prior sensitivity analysis}

The sensitivity of Bayesian results on the choice of priors has been discussed in many previous studies (Andrade and Teixeira, 2015; Gelman, 2006). For multilevel or other complex models, even the flat, uninformative uniform priors can become restrictive and informative on transformed parameters. Thus, it is important to test a diverse range of prior distributions and to see whether the same conclusion can be reached under different priors. In this study, priors are specified for the following parameters: the global intercept $\alpha$, the global age effect $\beta$, the global precision $\tau_{g}$ and precisions for street-level coefficients $\tau_{\xi}, \tau_{u}$ and $\tau_{v}$. As $\alpha, \beta$ and $\tau_{g}$ are global variables, there are sufficient amounts of data to lead to accurate posterior estimations (Gelman, 2006). So only $\tau_{\xi}$, $\tau_{u}$ and $\tau_{v}$ (in models NSP-2 and SP) are included in the sensitivity analysis.

Table 5 lists the prior combinations used in the sensitivity analysis. Gamma distribution is the usual choice of prior for

Table 5. Prior combinations in the sensitivity analysis

\begin{tabular}{|c|c|c|c|}
\hline Label & $\tau_{\xi}, \tau_{u}$ and $\tau_{v}$ & $\begin{array}{c}\text { DIC of } \\
\text { model NSP-2 }\end{array}$ & $\begin{array}{c}\text { DIC of } \\
\text { model SP }\end{array}$ \\
\hline A & Gamma(1, 0.0005) & 298193.9 & $297258 \cdot 1$ \\
\hline B & $\operatorname{Gamma}(0 \cdot 1,0 \cdot 1)$ & $297831 \cdot 0$ & $297384 \cdot 3$ \\
\hline C & $\operatorname{Gamma}(0 \cdot 01,0 \cdot 01)$ & $297890 \cdot 1$ & $297726 \cdot 3$ \\
\hline D & $\operatorname{Gamma}(0.001,0.001)$ & $298159 \cdot 3$ & 297474.0 \\
\hline$E$ & $\begin{array}{l}\text { Inverse truncated normal, } \\
\text { equivalent to the standard } \\
\text { deviation } \sigma=1 / \operatorname{sqrt}(\tau) \sim \\
\text { Normal }(0,0.01)\end{array}$ & $297972 \cdot 8$ & $297836 \cdot 7$ \\
\hline $\mathrm{F}$ & $\begin{array}{l}\text { Inverse truncated normal, } \\
\text { equivalent to the standard } \\
\text { deviation } \sigma=1 / \text { sqrt }(\tau) \sim \\
\text { Normal }(0,0.0001)\end{array}$ & $297850 \cdot 2$ & $298587 \cdot 7$ \\
\hline G & $\begin{array}{l}\text { Inverse truncated normal, } \\
\text { equivalent to the standard } \\
\text { deviation } \sigma=1 / \text { sqrt }(\tau) \sim \\
\text { Normal(10,0.0001) }\end{array}$ & $298500 \cdot 9$ & $297288 \cdot 5$ \\
\hline
\end{tabular}

$N(a, b)$ is normal distribution parameterised by mean $a$ and precision $b$. Gamma $(c, d)$ is Gamma distribution parameterised by shape $c$ and rate $d$ 


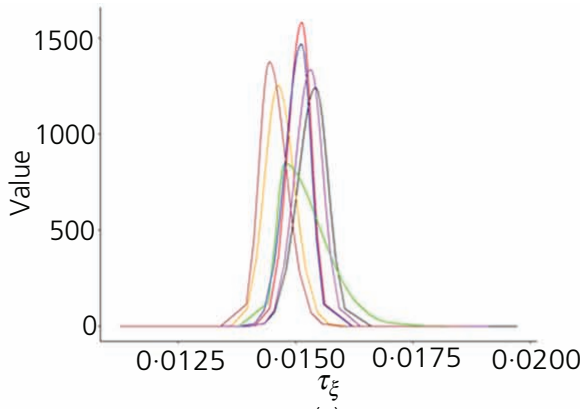

(a)

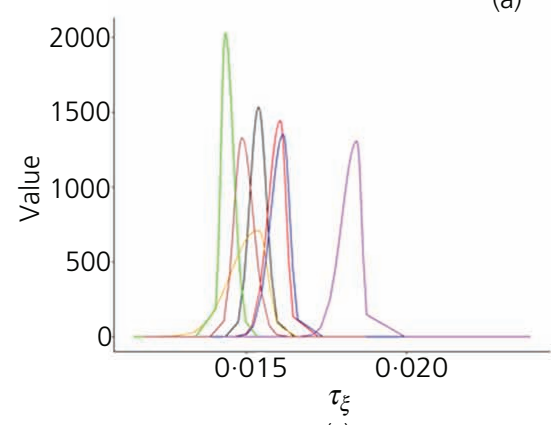

(c)

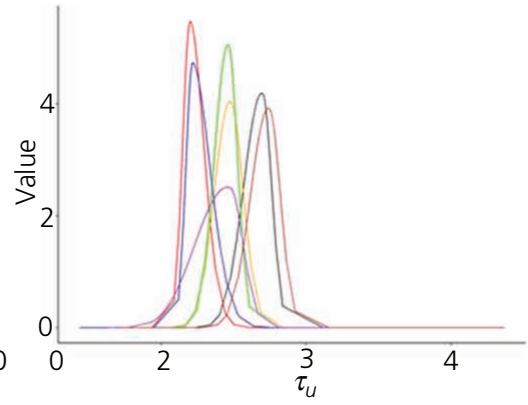

(b)

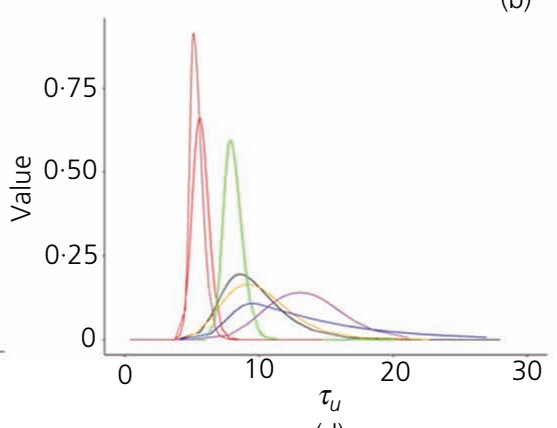

(d)

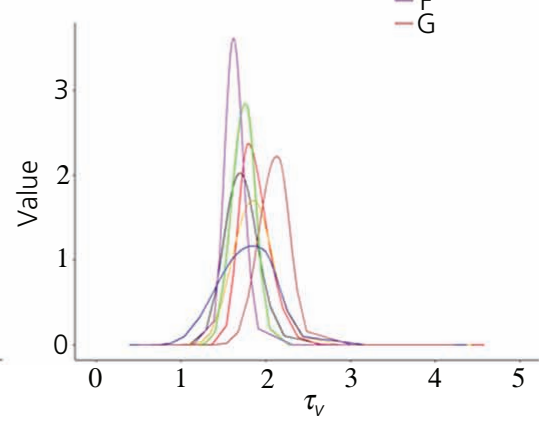

(e)

Figure 11. Posterior distributions under different priors: (a) $\tau_{\xi}$ of model NSP-2; (b) $\tau_{u}$ of model NSP-2; (c) $\tau_{\xi}$ of model SP; (d) $\tau_{u}$ of model $\mathrm{SP} ;(\mathrm{e}) \tau_{v}$ of model SP

precision parameters. Four Gamma priors with different distributional characters (labelled A, B, C and D) are tested first. It is possible to use other probability distributions as priors. However, as some streets only have two to three condition observations in the study period, uniform prior becomes too uninformative that the INLA process fails to complete (Ferkingstad and Rue, 2015). As a substitute, normal priors with large variances (labelled E, F and G) are tested instead.

Figure 11 shows the resulting posterior distributions of $\tau_{\xi}, \tau_{u}$ and $\tau_{v}$ under different prior specifications. Except for $\tau_{u}$ in model SP (Figure 11(c)), the posteriors do not differ much under different priors. For $\tau_{u}$ in model SP, although its mean and variance are sensitive to the prior specifications, its magnitude is almost always larger than $\tau_{v}$, implying that the spatial effect $v$ still has a larger variance $\left(1 / \tau_{v}\right)$, thus explaining the majority of the individual deviations in degradation rates. Figure 12 as well as the last two columns in Table 5 shows the DIC values for models NSP-2 and SP under different priors. Among the seven prior choices, the DIC of the spatial model SP is almost always smaller than the non-spatial model NSP-2, indicating the better performance of the spatial model in general.

\section{Discussions}

7.1 Limitations of the study

A weakness in this study, as shown Section 6, is that for all of the three models presented, their training and testing errors are

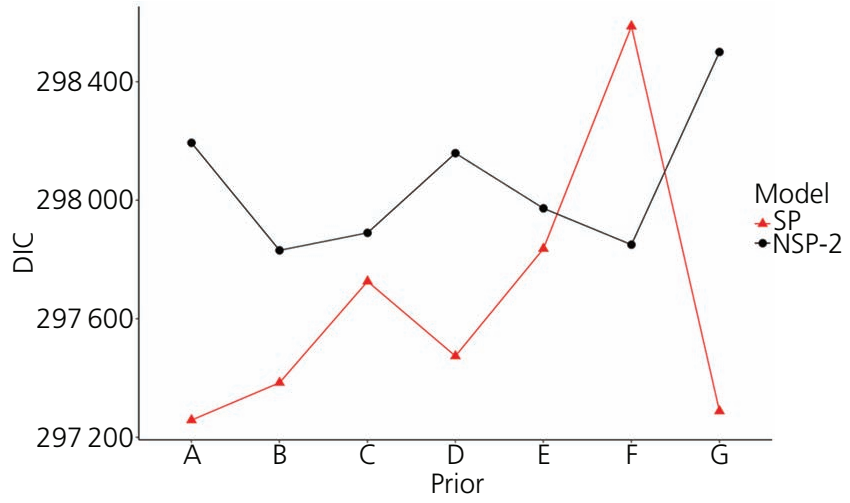

Figure 12. Resulting DIC of models NSP-2 and SP under different priors

around 10 PCI. This is possibly below the accuracy requirement for asset management. A limitation in the study is that degradations are only modelled linearly with age. Linear models are adopted because they show the key trends more directly than other complex models. However, the downside is that the model fit would be compromised. The PCI data used in this study are collected from visual surveys with large variabilities (Tan and Cheng, 2014). The large RMSEs in all three models are likely due to the fact that their simple forms cannot fit the variability (or errors) in the measurement. Nevertheless, the 
models are still sufficient to demonstrate the advantages of the spatial model.

\subsection{Advantages of the spatial model}

For this case study of modelling pavement degradation in San Francisco, the spatial model wins only by a slender lead in terms of accuracy. However, the real advantages of the spatial model are within the analysis: first of all, it is able to estimate the degradation parameters for road sections with missing or erroneous observations by borrowing information from adjacent sections, while the richness in the spatial information is lost in non-spatial models. Moreover, it can visually illustrate regions where pavements degrade faster than average. These regions do not necessarily have the worst pavement condition, but they may need maintenance more often in the long term. Local engineers can be consulted, or site investigations conducted, as for the underlying causes. The latter makes spatial models particularly useful in the real practice, as it can assist asset managers to narrow down their attention to a smaller region.

\subsection{Network-wide understanding and smart infrastructure management}

The spatial pavement degradation model proposed in this study is closely related to the recent advances in the field of smart infrastructure and management. The spatial modelling is built on two decades of continuous records of city-scale pavement condition data. Such input data are premised on advanced sensing and digital data inventory technologies for pavement infrastructures. On the other hand, the spatial pavement degradation model is also an example of how interdisciplinary data analysis techniques can contribute to the management of smart infrastructures. As a basis, it addresses the imperfections (measurement errors and missing predictors) in pavement condition data and identifies critical regions where pavements tend to age faster. Such results can promote local engineers to conduct more informed inspections/site investigations, eventually making more effective asset management decisions.

\section{Future work}

In the future work, more sophisticated spatio-temporal models will be experimented on the pavement degradation data set. Specifically, convolutional long short-term memory has shown promising performance in modelling spatio-temporal events, such as precipitation nowcasting and taxi demand forecasting (Xingiian et al., 2015; Yao et al., 2018). These experiments will be carried out to test their abilities in reducing the modelling errors.

Also, the model evaluation metrics used in this current study (RMSE and DIC) do not fully reflect the need in the real pavement management practice. Other metrics, such as asymmetric loss functions to account for overpredicting and underpredicting, will be incorporated.

The application of a spatial model will be further illustrated with similar case studies. Acknowledging that pavement degradation is a spatial process, future case studies will explore whether focusing and investing in critical pavement sections identified by spatial models will achieve cost benefits and serve to improve future pavement conditions in the long run.

Furthermore, the current study on degradation modelling will be extended into asset management and decision-making. This will be done through a series of simulation experiments that link pavement degradation with a city-scale traffic model, in order to study the feedback effect of different maintenance strategies.

\section{Summary}

This paper incorporates the concept of spatial dependency to the analysis of the ageing of pavements. A spatial model is proposed where the pavement degradation rate of a street segment is determined by its degradation history as well as the degradation history of its neighbouring segments. Two non-spatial models are also included for comparison. The approximation-based, efficient Bayesian INLA method is used for parameter inference. Built on more than two decades of pavement condition survey data collected from the San Francisco road network, it is found that the spatial model is more preferred than the non-spatial models from a Bayesian model selection perspective. The most important advantage of the spatial model is to reveal regions where streets with high or low degradation rates cluster. However, it is still necessary for the spatial modelling methodology to be tested on a wider variety of data sets and scenarios, as well as to reduce the modelling errors by incorporating more features or non-linear effects. The spatial models can contribute to the improvements of degradation modelling by extracting out the spatially correlated effects, which is likely to be caused by spatially correlated factors such as microclimate, traffic and material continuity. The improved pavement degradation model can then be utilised to organise targeted inspections, to investigate the underlying causes of degradation in vulnerable regions and to benefit asset management activities by supporting system-level maintenance planning.

\section{Acknowledgements}

The authors would like to thank Dr Ruchi Choudhary, Gerard Casey and Dr Krishna Kumar for their help in this work. Also, the authors would like to thank the San Francisco Department of Public Works and DataSF for making the pavement condition data available and the R-INLA project for providing the efficient analysis tools.

\section{REFERENCES}

Alaswadko N, Hassan R, Meyer D and Mohammed B (2019) Modelling roughness progression of sealed granular pavements: a new approach. International Journal of Pavement Engineering 20(2): 222-232, https://doi.org/10.1080/10298436.2017.1283689.

Aljoufie M, Zuidgeest M, Brussel M, van Vliet J and van Maarse-veen M (2013) A cellular automata-based land use and transport interaction model applied to Jeddah, Saudi Arabia. Landscape and Urban Planning 112: 89-99, https://doi.org/10.1016/j.landurbplan.2013.01. 003.

Andrade AR and Teixeira PF (2015) Statistical modelling of railway track geometry degradation using hierarchical Bayesian models. Reliability 
Engineering \& System Safety 142: 169-183, https://doi.org/10.1016/j. ress.2015.05.009

Anyala M, Odoki J and Baker C (2014) Hierarchical asphalt pavement deterioration model for climate impact studies. International Journal of Pavement Engineering 15(3): 251-266, https://doi.org/10.1080/ 10298436.2012.687105.

Baller RD, Anselin L, Messner SF, Deane G and Hawkins DF (2001) Structural covariates of U.S. county homicide rates: incorporating spatial effects. Criminology 39(3): 561-588, https://doi.org/10.1111/j. 1745-9125.2001.tb00933.x

Benbow E, Wright A, Dhillon N, Harrington M and Nesnas K (2017) Development of SCANNER and UKPMS: Task 1 - Consistency of SCANNER Data and Task 2 - SCANNER Condition Parameters. Transport Research Laboratory Limited, Berks, UK, Technical Report PPR816.

Besag J, York J and Mollié A (1991) Bayesian image restoration, with two applications in spatial statistics. Annals of the Institute of Statistical Mathematics 43(1): 1-20, https://doi.org/10.1007/BF00116466.

Blangiardo M and Cameletti M (2015) Bayesian computing. In Spatial and Spatio-temporal Bayesian Models with R-INLA. Wiley, Chichester, UK, pp. 75-125.

Blangiardo M, Cameletti M, Baio G and Rue H (2013) Spatial and spatiotemporal models with R-INLA. Spatial and Spatio-temporal Epidemiology 4: 33-49, https://doi.org/10.1016/j.sste.2012.12.001.

Chen C, Zhang S, Zhang G, Bogus SM and Valentin V (2014) Discovering temporal and spatial patterns and characteristics of pavement distress condition data on major corridors in New Mexico. Journal of Transport Geography 38: 148-158, https://doi.org/10.1016/j.jtrangeo. 2014.06.005

Croissant Y and Millo G (2008) Panel data econometrics in R: the plm package. Journal of Statistical Software 27(2): 1-43, https://doi.org/ 10.18637/jss.v027.i02.

DataSF (2019) http://data.sfgov.org (accessed 11/01/2019).

Deshmukh MM (2010) Development of Equations to Determine the Increase in Pavement Condition due to Treatment and the Rate of Decrease in Condition after Treatment for a Local Agency Pavement Network. Master's thesis, Texas A\&M University, College Station, TX, USA. See http://hdl.handle.net/1969.1/ETD-TAMU-2009-05-317 (accessed 11/01/2019).

Dormann CF, McPherson JM, Arajo MB et al. (2007) Methods to account for spatial autocorrelation in the analysis of species distributional data: a review. Ecography 30(5): 609-628, https://doi.org/10.1111/j.2007. 0906-7590.05171.x.

Ferkingstad E and Rue H (2015) Improving the INLA approach for approximate Bayesian inference for latent Gaussian models. Electronic Journal of Statistics 9(2): 2706-2731, https://doi.org/10.1214/15EJS1092.

Ferreira A, Picado-Santos L and Antunes A (2002) A segment-linked optimization model for deterministic pavement management systems. International Journal of Pavement Engineering 3(2): 95-105, http:// dx.doi.org/10.1080/10298430290030603.

Ferreira A, de Picado-Santos L, Wu Z and Flintsch G (2011) Selection of pavement performance models for use in the Portuguese PMS International Journal of Pavement Engineering 12(1): 87-97, https:// doi.org/10.1080/10298436.2010.506538.

Gao L and Zhang Z (2012) Approximation approach to problem of large-scale pavement maintenance and rehabilitation. Transportation Research Record 2304: 112-118, http://dx.doi.org/10. 3141/2304-13.

Gelman A (2006) Prior distributions for variance parameters in hierarchical models (comment on article by Browne and Draper). Bayesian Analysis 1(3): 515-534, https://doi.org/10.1214/06-BA117A

Gelman A and Hill J (2006) Data Analysis Using Regression and Multilevel/Hierarchical Models. Cambridge University Press, Cambridge, UK.
Golabi K, Kulkarni RB and Way GB (1982) A statewide pavemen management system. Interfaces 12(6): 5-21, http://dx.doi.org/10.1287/ inte.12.6.5.

Haider SW, Baladi GY, Chatti K and Dean CM (2010) Effect of frequency of pavement condition data collection on performance prediction. Transportation Research Record 2153: 67-80, http://dx.doi.org/10. 3141/2153-08.

Handcock MS and Wallis JR (1994) An approach to statistical spatialtemporal modeling of meteorological fields. Journal of the American Statistical Association 89(426): 368-378, https://doi.org/10.2307/ 2290832.

Jahanbakhsh S, Gao L and Zhang Z (2016) Estimating Spatial Dependence Associated with Deterioration Process of Road Network. Transport Research Board, Washington, DC, USA, Technical Report 16-6179.

Johnson KD and Cation KA (1992) Performance prediction development using three indexes for North Dakota pavement management system. Transportation Research Record (1344): 22-30.

Lavine ML and Hodges JS (2012) On rigorous specification of ICAR models. The American Statistician 66(1): 42-49, https://doi.org/10. 1080/00031305.2012.654746.

Lichstein JW, Simons TR, Shriner SA and Franzreb KE (2002) Spatial autocorrelation and autoregressive models in ecology. Ecological Monographs 72(3): 445-463, https://doi.org/10.1890/0012-9615(2002) 072[0445:SAAAMI]2.0.CO;2.

McQueen J and Timm D (2005) Part 2: pavement monitoring, evaluation, and data storage: statistical analysis of automated versus manual pavement condition surveys. Transportation Research Record 1940 $53-62$.

Morosiuk G, Riley M and Odoki J (2004) Modelling Road Deterioration and Works Effects in HDM-4. World Road Association and World Bank, Paris, France and Washington, DC, USA. See http://www.lpcb. org/index.php/documents/pavement-deterioration/25191-2004-hdm4pavement-deterioration-and-maintenance-effects-modelling (accessed 22/07/2015).

Nunez MM and Shahin MY (1986) Pavement condition data analysis and modeling. Transportation Research Record 1070: 125-132.

ODC (Open Data Commons) (2009) ODC Public Domain Dedication and Licence (PDDL). ODC, Cambridge, UK. See https://opendatacommons. org/licenses/pddl/1.0/index.html (accessed 21/01/2019).

Ortiz-García JJ, Costello SB and Snaith MS (2006) Derivation of transition probability matrices for pavement deterioration modeling. Journal of Transportation Engineering 132(2): 141-161, https://doi.org/10.1061/ (ASCE)0733-947X(2006)132:2(141).

Paterson WD (1987) Road Deterioration and Maintenance Effects: Models for Planning and Management. Johns Hopkins University Press, Baltimore, MD, USA. See http://documents.worldbank.org/ curated/en/222951468765265396/Road-deterioration-and-maintenanceeffects-models-for-planning-and-management (accessed 11/01/2019).

R Core Team (2017) R: a Language and Environment for Statistical Computing. R Foundation for Statistical Computing, Vienna, Austria. See https://www.R-project.org/ (accessed 11/01/2019).

R-INLA Project (2019) http://www.r-inla.org (accessed 11/01/2019)

Ramirez-Flores RA and Chang-Albitres C (2012) A Stochastic Approach for Pavement Condition Projections and Budget Needs for the MTC Pavement Management System. Transportation Research Board, Washington, DC, USA. See http://onlinepubs.trb.org/onlinepubs/ conferences/2012/assetmgmt/presentations/Data-A-Ramirez-FloresChang-Albitres.pdf (accessed 11/01/2019).

Rue $\mathrm{H}$ and Martino S (2007) Approximate Bayesian inference for hierarchical Gaussian Markov random field models. Journal of Statistical Planning and Inference 137(10): 3177-3192, https://doi.org/ 10.1016/j.jspi.2006.07.016.

Rue H, Martino S and Chopin N (2009) Approximate Bayesian inference for latent Gaussian models by using integrated nested Laplace approximations. Journal of the Royal Statistical Society: Series B 
Smart Infrastructure and Construction

Volume 171 Issue SC3
Pavement degradation: a city-scale model

for San Francisco, USA

Zhao, Silva and Soga
(Statistical Methodology) 71(2): 319-392, https://doi.org/10.1111/j. 1467-9868.2008.00700.x

Sadeghi J, Najafabadi ER and Kaboli M (2017) Development of degradation model for urban asphalt pavement. International Journal of Pavement Engineering 18(8): 659-667, https://doi.org/10.1080/ 10298436.2015.1095912.

Saliminejad S and Gharaibeh NG (2012) A spatial-Bayesian technique for imputing pavement network repair data. Computer-aided Civil and Infrastructure Engineering 27(8): 594-607, https://doi.org/10.1111/j. 1467-8667.2012.00762.x.

Schrödle B and Held L (2011) A primer on disease mapping and ecological regression using INLA. Computational Statistics 26(2): 241-258, https://doi.org/10.1007/s00180-010-0208-2.

SF Public Works (San Francisco Public Works) (2017) Press Release: SF Street Pavement Condition Improves for 5th Year in a Row - 1/3/2017. SF Public Works, San Francisco, CA, USA. See https://sfpublicworks. org/project/press-release-sf-street-pavement-condition-improves-5thyear-row-132017 (accessed 11/01/2019).

Shahin MY and Kohn SD (1982) Overview of the 'PAVER' Pavement Management System and Economic Analysis of Field Implementing the 'PAVER' Pavement Management System. Army Construction Engineering Research Laboratory, Champaign, IL, USA. See http:// www.dtic.mil/cgi-bin/GetTRDoc?Location=U2\&doc=GetTRDoc pdf\&AD=ADA116311 (accessed 11/01/2019).

Shahin MY, Darter MI and Kohn SD (1978) Development of a Pavement Condition Index for Roads and Streets. Army Construction Engineering Research Laboratory, Champaign, IL, USA, Technical Report ADA057148. See http:/www.dtic.mil/dtic/tr/fulltext/u2/ a057148.pdf (accessed 11/01/2019).

Silva EA and Clarke KC (2005) Complexity, emergence and cellular urban models: lessons learned from applying SLEUTH to two Portuguese metropolitan areas. European Planning Studies 13(1): 93-115, https:// doi.org/10.1080/0965431042000312424.

Spiegelhalter DJ, Best NG, Carlin BP and Van Der Linde A (2002) Bayesian measures of model complexity and fit. Journal of the Royal Statistical Society: Series B (Statistical Methodology) 64(4): 583-639, https://doi.org/10.1111/1467-9868.00353.

Spiegelhalter DJ, Best NG, Carlin BP and van der Linde A (2014) The deviance information criterion: 12 years on. Journal of the Royal
Statistical Society: Series B (Statistical Methodology) 76(3): 485-493, https://doi.org/10.1111/rssb.12062.

Spong C and Cartwright R (2017) Development of SCANNER and UKPMS: Task 3 - Appropriateness of the SCANNER RCI. Transport Research Laboratory Limited, Berks, UK, Technical Report PPR817.

Tan SG and Cheng D (2014) Quality assurance of performance data for pavement management systems. In Design, Analysis, and Asphalt Material Characterization for Road and Airfield Pavements (Lee JL Hossain Z and $\mathrm{Hu} \mathrm{X}$ (eds)). American Society of Civil Engineers, Reston, VA, USA, pp. 163-169.

Tierney L and Kadane JB (1986) Accurate approximations for posterior moments and marginal densities. Journal of the American Statistical Association 81(393): 82-86, https://doi.org/10.1080/01621459.1986. 10478240 .

Vital Signs (2017) Street Pavement Condition. Vital Signs, San Francisco, CA, USA. See http://www.vitalsigns.mtc.ca.gov/street-pavementcondition (accessed 11/01/2019).

Wang G, Frith D and Morian D (2014) Implementation of PMS at a local level-case study based on StreetSaver ${ }^{\circledR}$. In Civil Engineering and Urban Planning III (Mohammadian K, Goulias K, Cicek E, Wang JJ and Maraveas C (eds)). Taylor \& Francis, London, UK, pp. 207-212.

Wu Z, Flintsch G W and Chowdhury T (2008) Hybrid multi-objective optimization model for regional pavement-preservation resource allocation. Transportation Research Record 2084: 28-37.

Xingjian S, Chen Z, Wang H et al. (2015) Convolutional LSTM network: a machine learning approach for precipitation nowcasting. Proceedings of Advances in Neural Information Processing Systems 28 (NIPS 2015), Montreal, QC, Canada, pp. 802-810.

Yao H, Wu, F, Ke J et al. (2018) Deep Multi-view Spatial-Temporal Network for Taxi Demand Prediction. arXiv, Ithaca, NY, USA, arXivpreprint arXiv:1802.08714.

Zhang H, Keoleian GA and Lepech MD (2012) Network-level pavement asset management system integrated with life-cycle analysis and lifecycle optimization. Journal of Infrastructure Systems 19(1): 99-107, http://dx.doi.org/10.1061/(ASCE)IS.1943-555X.0000093.

Zhao B and Nagayama T (2017) IRI estimation by the frequency domain analysis of vehicle dynamic responses. Procedia Engineering 188 9-16, https://doi.org/10.1016/j.proeng.2017.04.451.

\section{How can you contribute?}

To discuss this paper, please email up to 500 words to the editor at journals@ice.org.uk. Your contribution will be forwarded to the author(s) for a reply and, if considered appropriate by the editorial board, it will be published as discussion in a future issue of the journal.

Proceedings journals rely entirely on contributions from the civil engineering profession (and allied disciplines). Information about how to submit your paper online is available at www.icevirtuallibrary.com/page/authors, where you will also find detailed author guidelines. 